\title{
m6A Methylation Regulators Are Predictive Biomarkers for Tumour Metastasis in Prostate Cancer
}

\section{Xiaobo Wu}

Huashan Hospital Fudan University

Qianwen Ge

Huashan Hospital Fudan University

Chen Yang

Huashan Hospital Fudan University

Yishuo Wu

Huashan Hospital Fudan University

Mengbo Hu

Huashan Hospital Fudan University

Chenkai Ma ( $\nabla$ dr.ckma@gmail.com )

CSIRO https://orcid.org/0000-0003-2172-7332

Haowen Jiang

Huashan Hospital Fudan University

Jimeng Hu

Huashan Hospital Fudan University

\section{Primary research}

Keywords: Prostate cancer, metastasis, biomarker, m6A, methylation, immune cell infiltration

Posted Date: October 23rd, 2020

DOI: https://doi.org/10.21203/rs.3.rs-95052/v1

License: (1) (1) This work is licensed under a Creative Commons Attribution 4.0 International License. Read Full License 


\section{Abstract}

\section{Background}

Prostate cancer is one of the most common cancers in men. Usually, most prostate cancers are localized in initial diagnoses and grow slowly. Patients with localized prostate cancers have a nearly $100 \% 5$-year survival rate; however, the 5-year survival rate of metastatic or progressive prostate cancer is still dismal. N6-methyladenosine ( $\mathrm{m} 6 \mathrm{~A}$ ) is the most common post-transcriptional mRNA modification and is dynamically regulated by $\mathrm{m} 6 \mathrm{~A}$ regulators. A few studies have shown that the abnormal expression of m6A regulators is significantly associated with cancer progression and immune cell infiltration, but the roles of these regulators in prostate cancer remain unclear.

Methods

Here, we comprehensively examined the patterns of 21 m6A regulators across 494 prostate cancers and systematically correlated $\mathrm{m} 6 \mathrm{~A}$ regulators with prostate cancer progression and immune cell infiltration. Consensus clustering was utilized for the subtype identification of m6A regulators for prostate cancers. Each subtype signature genes were obtained by the pairwise differentially expressed genes. Featured pathways of m6A subtypes were predicted consequently. The m6A score was constructed to predict the m6A activation. The association of m6A score with patients' survival, metastasis and immune cell infiltration were also investigated.

Results

We identified three distinct clusters in prostate cancer based on the expression profiles of $21 \mathrm{~m} 6 \mathrm{~A}$ regulators by consensus clustering. The differential expression and pathway analyses on the three clusters uncovered the m6A regulators involved in metabolic processes and immune responses in prostate cancer. Moreover, we established an m6A score to perceive the m6A regulator activation for prostate cancer. The m6A score is significantly associated with Gleason scores and metastasis in prostate cancer. The predictive capacity of m6A score on prostate cancer metastasis was also validated in another independent cohort.

Conclusion

Our study revealed the critical role of $\mathrm{m} 6 \mathrm{~A}$ regulators in prostate cancer progression and $\mathrm{m} 6 \mathrm{~A}$ score is promising predictive biomarker for prostate cancer metastasis.

\section{Introduction}

Prostate cancer is the most common tumour in men. Although prostate cancer is usually benign and localized in the initial diagnosis, metastasis is still the most frequent cause for prostate cancer-related mortality $(1,2)$. Metastatic prostate cancer is responsible for more than $90 \%$ of deaths in patients with prostate cancer. The rapid growth and progression of metastatic prostate cancer is largely driven by 
androgen; therefore, androgen-deprivation therapy (ADT) is one of the first-line treatments for symptomatic metastatic prostate cancer (3). However, the duration of response of ADT is variable, and resistance is inevitable in metastatic prostate cancer after ADT. The outcomes for metastatic castrationresistant prostate cancer (mCRPC) are still dismal, though a few attempts and new regimens have been investigated to improve their efficacy for mCRPC (4-7). Therefore, a further understanding of the driving force and regulatory molecules in MCRPC is key to improve the outcomes of patients with mCRPC.

Previous studies have uncovered a few potential mechanisms of resistance in mCRPC independent of driver mutation status. ADT impairs androgen receptor (AR) signalling-dependent cell growth in prostate cancer by the reduction of secretory androgen (8). However, the AR signalling is likely restored after a few periods of ADT. In addition, ADT can introduce the bypass of AR signalling by activating other receptor signalling pathways. AR-independent signalling also highlights the heterogeneity of mCRPC with regard to gene expression and signalling transduction (9). Hence, further investigation on the regulatory mechanism beyond the AR signalling and the relationship of heterogeneity and metastatic phenotype in prostate cancer will precisely stratify patients according to their metastatic risk.

N6-methyladenosine (m6A) is the most prominent RNA modification regulating the transcription, stabilization and translocation of RNA without DNA or RNA base changes (10). Similar to DNA methylation, $\mathrm{m} 6 \mathrm{~A}$ is a biologically reversible process that is elaborately regulated by methyltransferases, demethylases and binding proteins, also known as "writers", "erasers" and "readers", respectively (11). The methylation marks of m6A are dynamically catalysed by methyltransferases, usually consisting of RBM15, ZC3H13, METTL3, METTL14 and WTAP, and removed by demethylases, such as FTO and ALKBH5. The RNA-binding proteins ("readers") YTHDF1/2/3, YTHDC1/2, HNRNPA2B1, LRPPRC and FMR1 can recognize the m6A status in RNAs and mediate the biological functions of these RNAs. Accumulating evidence has demonstrated that $\mathrm{m} 6 \mathrm{~A}$ modification is involved in multiple biological processes. Neural-simulated memory learning is facilitated by m6A regulators $(12,13)$. A decrease in pluripotency is also observed in stem cells with high activation of m6A regulators (14). Inactivation of m6A regulators is associated with cancer metastasis in the liver, colon, kidney and pancreas (15-18). In prostate cancer, high expression of METTL3 elevates the growth and progression of cancer cells. However, the biological functions of other m6A regulators and the comprehensive landscape of $\mathrm{m} 6 \mathrm{~A}$ regulators still remain unclear in prostate cancer. Moreover, the subtypes of cancer stratified by m6A regulators have distinct prognoses, indicating that $\mathrm{m} 6 \mathrm{~A}$ regulators likely contribute to the heterogeneity of mCRPC. The metastatic risk of prostate cancer could be inferred by the expression of m6A regulators.

Here, we utilized the RNA sequencing data of prostate cancer to establish the comprehensive expression patterns of m6A regulators. We identified differentially expressed genes between subtypes with distinct levels of $\mathrm{m} 6 \mathrm{~A}$ regulators that were enriched in immune response and metabolic regulation. Immune cell infiltration was also associated with $\mathrm{m} 6 \mathrm{~A}$ regulator expression patterns. For application of metastatic prediction by m6A regulator patterns, we constructed an m6A score. This m6A score demonstrated a moderate prediction for prostate cancer metastasis. 


\section{Methods}

\section{Data pre-processing}

Public gene expression data and full clinical annotations of prostate adenocarcinoma (PRAD) were downloaded from the Cancer Genome Atlas (TCGA) database. RNA sequencing data (FPKM value) of gene expression and DNA methylation profiles by Illumina 450K were downloaded from the Genomic Data Commons (GDC, ttps://portal.gdc.cancer.gov/). The mutation files with annotations of corresponding samples from TCGA PRAD were downloaded from firehose (https://gdac.broadinstitute.org/). The CpG loci were annotated by the package "IlluminaHumanMethylation450kanno.ilmn12.hg19". Batch effects from non-biological technical biases were removed using the "ComBat" algorithm of the sva package. The somatic mutation data of TCGA PRAD were acquired from the firehose database (https://gdac.broadinstitute.org/).

\section{Consensus clustering}

An unsupervised consensus clustering of $21 \mathrm{~m} 6 \mathrm{~A}$ regulators was utilized to investigate the subtypes of prostate cancer. The m6A regulators included 8 writers (METTL3, METTL14, RBM15, RBM15B, WTAP, KIAA1429, CBLL1, ZC3H13), 2 erasers (ALKBH5, FTO) and 11 readers (YTHDC1, YTHDC2, YTHDF1, YTHDF2, YTHDF3, IGF2BP1, HNRNPA2B1, HNRNPC, FMR1, LRPPRC, ELAVL1). First, we performed the Student $t$-test to select the differentially expressed m6A regulators between prostate cancer and normal prostate tissue. We only selected the significantly expressed m6A regulators for downstream analysis. Then, we applied hierarchical clustering with agglomerative average linkage to detect robust clusters in prostate cancer. The distance metric was 1-(Pearson's correlation coefficient) and was run over 1000 iterations. SigClust was performed to establish the significance of each cluster in a pairwise matter. Silhouette width is defined as the ratio of each sample's average distance to samples in the same cluster to the smallest distance to samples not in the same cluster. The best number of cluster group is determined by the Cumulative Distribution Function (CDF) curve and the delta change of CDF in each subtype number.

\section{Differentially expressed gene (DEG) analysis}

The differentially expressed genes between subtypes were identified by the "limma" package in a pairwise fashion. The significance was defined as log fold change larger than 2 and FDR less than 0.05 . The list of DEGs was determined after removal of the replicated genes in each comparison.

\section{Pathway and immune cell prediction}

The enriched functional pathway of DEG was annotated by Metascape (https://metascape.org/gp/index.html) in the express module. P-values were calculated based on the cumulative hypergeometric distribution, while q-values were calculated using the Benjamini-Hochberg procedure to account for multiple testings. Pathway terms with $p$-values $<0.01$, minimum counts of 3 , and enrichment factors $>1.5$ (the enrichment factor is the ratio between the observed counts and the 
counts expected by chance) were collected and grouped into clusters based on their membership similarities. PaGenBase was utilized for the tissue specificity of DEGs. The Gene Set Enrichment Analysis (GSEA) was conducted in GSEA 4.0.2 for identification of enriched pathway using pairwise fashion. The immune cell infiltration in prostate cancer was predicted by CIBERSORT (https://cibersort.stanford.edu/) with 100 permutation runs.

\section{M6A score}

To quantify the m6A regulator patterns of individual samples, we constructed a set of scoring systems to evaluate the m6A regulator levels of individual patients with prostate cancer. The m6A score was established according to a previous study with some modifications (19). In brief, first, we filtered out m6A regulators that were not differentially expressed between prostate cancer and normal prostate tissue. Second, we utilized the Student's $t$ test to identify the m6A signatures associated with prostate cancer metastasis and only took the differentially expressed m6A regulators between metastatic prostate cancer and non-metastatic prostate cancer into the establishment of the m6A score. We then performed principal component analysis (PCA) to construct m6A regulator signature patterns. Principal components 1 and 2 were both selected to form the m6A score (Supplementary figure 1).

\section{m6A score $=\sum P C 1 i+P C 2 i$}

where $i$ is the prostate cancer metastasis-related signature gene.

\section{Stemness Index}

The Stemness Index of each sample predicted by an OCLR algorithm was described before. In brief, Malta et al established a predictive model on pluripotent stem cell samples to train a stemness signature (20). A gene expression profile containing 24 genes was included in the mRNA expression-based signature. We applied the stemness index model to score the prostate cancer samples by RNA expression data. The stemness index was subsequently scaled to the $[0,1]$ range by subtracting the minimum and dividing by the maximum.

\section{Statistical analysis}

Correlation coefficients between the m6A score and the expression of m6A regulators, as well as Stemness, were determined by Spearman and distance correlation analyses. One-way ANOVA was used to conduct difference comparisons of three or more groups. The overall survival (OS) and recurrence-free survival (RFS) of each m6A subtype were compared to investigate the correlation of m6A subtypes and patient outcomes. The survival curves for the survival analysis were generated via the Kaplan-Meier method, and log-rank tests were utilized to identify the significance of differences between subtypes. The specificity and sensitivity of m6Ascore and SNPH were assessed through receiver operating characteristic (ROC) curve. The area under the curve (AUC) was quantified and plotted using the pROC R package. The 
waterfall function of the maftools package was used to present the mutation landscape of all $\mathrm{m} 6 \mathrm{~A}$ regulators in patients with prostate cancer from the TCGA cohort. For DNA methylation levels, the CpG loci located in promoter areas (genomic location: TSS500 or TSS1500) of m6A regulators were utilized for comparison between m6A subtypes. All statistical $P$ values were two-sided, with $p<0.05$ as statistically significant. All data processing was performed in R 3.6.0 software.

\section{Results}

\section{Consensus clustering of m6A regulators suggested three distinct subtypes in prostate cancer.}

To investigate the similarity and discrepancy of m6A regulator expression patterns between prostate cancers, we first performed consensus clustering on 494 prostate cancer samples from the TCGA PRAD dataset using 21 pre-defined m6A regulators (METTL3, METTL14, RBM15, RBM15B, WTAP, KIAA1429, CBLL1, ZC3H13, ALKBH5, FTO, YTHDC1, YTHDC2, YTHDF1, YTHDF2, YTHDF3, IGF2BP1, HNRNPA2B1, HNRNPC, FMR1, LRPPRC, ELAVL1). First, 18 of 21 m6A regulators (METTL3, METTL14, RBM15B, KIAA1429, CBLL1, ZC3H13, ALKBH5, FTO, YTHDC1, YTHDC2, YTHDF1, YTHDF2, IGF2BP1, HNRNPA2B1, HNRNPC, FMR1, LRPPRC, ELAVL1) were identified as differentially expressed in prostate cancer as compared to normal prostate tissue (supplementary figure $3 \mathrm{~B}$ ), suggesting that most m6A regulators were biologically pro-active in prostate cancer. Therefore, we selected these $18 \mathrm{~m} 6 \mathrm{~A}$ regulators as key m6A regulators for consensus clustering. Consensus average linkage hierarchical clustering identified three robust subtypes $(k=3)$ with a significant increase of clustering stability from $k=2$ to 6 (Figure $1 \mathrm{~A}$, Supplementary figure 2). As we observed a marginal increase of clustering stability after $k=3$ (less than 0.2) (Supplementary figure 2), we clustered and defined these three consensus clusters as Subtype 1, Subtype 2 and Subtype 3. Cluster significance was evaluated by SigClust. The boundary between Subtype 1 and Subtype 2 was statistically significant, but no significance was observed between Subtype 1 versus Subtype 3 and Subtype 2 versus Subtype 3, suggesting that Subtype 3 is likely an intermediate or overlapping status between Subtype 1 and Subtype 2 (Supplementary figure 2). Principal Component Analysis (PCA) also revealed a separation of Subtype 1 and Subtype 2 and an overlap of Subtype 3 (Figure 1B). Samples in each subtype were identified based on their positive silhouette width, with few exceptions, suggesting that a higher similarity was observed to their own cluster than to any other clusters (Figure 1C). We also observed a difference of m6A regulator expression between subtypes

(Figure 1D), where half of the m6A regulators were highly expressed in Subtype 1 and Subtype 2. Notably, almost all m6A regulators were highly expressed in Subtype 3, suggesting an extra-active m6A modification likely occurred in Subtype 3 of prostate cancer.

\section{Different immune responses and the activation of metabolic pathways were observed in m6A regulator subtypes of prostate cancer}

Since prostate cancer revealed three distinct subtypes in the expression of m6A regulators, we further explored the differentially expressed genes between subtypes to uncover the key pathways mediated by m6A regulators. A pairwise differentially expressed gene (DEG) analysis identified 24 genes that were 
significantly expressed between the three m6A subtypes (Supplementary figure 3A, Supplementary Table 1), suggesting the mRNAs from these 24 genes were likely modified or co-regulated by m6A regulators. It is notable that all samples clustered in Subtype 3 had intermediate or high risk of Gleason scores, while Subtype 1 and 2 had mixtures of prostate cancer patients with low, intermediate and high risks of Gleason scores (Supplementary figure 3A), suggesting that m6A regulation likely occurred in all stages of prostate cancer. Through the Gene Otology and pathway analysis in Metascape, we found that these differentially expressed genes were enriched in metabolic processes and immune response (Figure 2A). As advanced prostate cancer demonstrated an overwhelming de novo resistance to immune checkpoint blockade (21), an investigation on the relationship of m6A regulators and immune cell infiltration probably promoted the efficacy of immune checkpoint blockade in prostate cancer. To explore the immune cell infiltration patterns in each subtype, we performed a prediction for immune cell proportions by CIBERSORT. Notably, the levels of CD8+ T cells and activated dendritic cells were markedly different in each subtype (ANOVA, $p<0.001$ and $p=0.001$, respectively) (Figure 2B), suggesting there was m6A regulation involved in immune cell infiltration in prostate cancer. To further validate the enriched key pathways between subtypes, we then performed the Gene Set Enrichment Analysis (GSEA) on 454 PCa samples. The GSEA confirmed that the Oxidative phosphorylation and Fatty acid metabolism were enriched in the Subtype $1 \mathrm{PCa}$ while the inflammatory response pathway was downregulated in the Subtype 3 (supplementary figure 4).

\section{Clustering of m6A regulators was associated with metastasis in prostate cancer}

To test whether the clustering of m6A regulators was associated with prostate patient outcomes, we compared the survivals in each subtype. Interestingly, there were no significant differences in overall and recurrence-free survival between m6A subtypes (Figure 3A), suggesting that prostate cancer recurrence was likely not modulated by $\mathrm{m} 6 \mathrm{~A}$ regulators. Considering the facts that a few m6A regulators were different between 55 normal prostate tissue and 464 prostate cancer (Supplementary figure 3B) and prostate cancer metastasis was a more critical issue than recurrence, we then compared the m6A expression in the GSE147493 dataset, which included prostate cancers with metastasis or nonmetastasis. Seven m6A regulators (HNRNPA2B1, FMR1, METTL14, KIAA1429, YTHDF1, ALKBH5, HNRNPC) displayed different expression patterns between 62 metastatic and 37 non-metastatic prostate cancers (Figure 3B), suggesting that these seven m6A regulators were associated with prostate cancer metastasis. More importantly, we also observed that the subtypes clustered by m6A regulators were associated with Gleason Scores. No prostate cancer in Subtype 3 had tumour with a Gleason Score less than 7 (Supplementary figure 5A). Considering that cancer stem cells played a critical role in cancer initiation and the origin of cancer metastasis in prostate cancer, we then further tested the Stemness Index between subtypes. As we expected, Subtype 3 had a significantly highest Stemness Index of all three subtypes (Supplementary figure 5B). These results suggested that clustering of prostate cancer by the expression of m6A regulators was associated with prostate cancer metastasis.

\section{M6A score was a surrogate for m6A activation in prostate cancer.}


For generalization of m6A subtyping broadly and directly predict the metastasis of $\mathrm{PCa}$, we established an $\mathrm{m} 6 \mathrm{~A}$ score to quantify the activation of $\mathrm{m} 6 \mathrm{~A}$ in prostate cancer. Notably, seven m6A regulators were also differentially expressed between 464 prostate cancer and 55 normal prostate tissue (Supplementary figure 3B), suggesting these seven m6A regulators likely orchestrated the metastasis by activation of $\mathrm{m} 6 \mathrm{~A}$ in prostate cancer. Therefore, we took these seven m6A regulators as the key genes to investigate their expression in prostate cancer. The $\mathrm{m} 6 \mathrm{~A}$ score of each sample was the sum of the first and second $P C$ in the given sample. To test that the m6A scores we generated could surrogate the subtypes of prostate cancer, we first compared the m6A scores between subtypes. As expected, the three m6A subtypes had different expression of m6A scores, and Subtype 3 had the lowest level of m6A score among all subtypes (Figure 4A). The m6A score had an inverse correlation with most m6A regulators (Figure 4B), suggesting that the m6A score was likely a comprehensive surrogate for m6A inactivation in prostate cancer. In addition, m6A score also had a significant correlation with Gleason score (Figure 4C), suggesting m6A score could be a surrogate for PCa malignancy. A previous study revealed that the RNA demethylase ALKBH5 was selectively activated in cancer stem cells and promoted tumorigenesis in leukaemia; therefore, we tested whether our m6A score was associated with cancer stem cells in prostate cancer (22). To elucidate the concordance of m6A score and stemness in prostate cancer, we also inferred the proportion of cancer stem cells in prostate cancer by Stemness Index and found that the m6A score was significantly associated with Stemness Index $\left(r=-0.362, p=9 * 10^{-7}\right)$ (Figure 4D) (20). Taken together, these results suggested that the $\mathrm{m} 6 \mathrm{~A}$ score could be a promising predictive biomarker for prostate cancer metastasis.

To further validate the progression prediction for prostate cancer by m6A score, we compared the $\mathrm{m} 6 \mathrm{~A}$ score between prostate cancer with metastasis and non-metastasis. As expected, metastatic prostate cancers had lower levels of m6A scores than those in prostate cancer without metastasis (Figure 5A). Hence, we conducted a ROC to investigate the predictive capacity of m6A score on prostate cancer metastasis. The Area Under the Curve (AUC) of m6A score for the prediction of metastatic prostate cancer was 0.633 (Figure 5B). To further test the predictive effectiveness of $\mathrm{m} 6 \mathrm{~A}$ score, we then performed the m6A score for another independent cohort (GSE6919) that containing 25 metastastic PCa and 60 primary $\mathrm{PCa}$. As expected, our m6A score displayed a significant discrimination of metastatic PCa from primary PCa and the predictive accuracy in the validation cohort was 89.5\% (Figure 5C\&D). Our results demonstrated a superior diagnostic capacity of metastatic prostate cancer to SNPH (Supplementary figure 6), suggesting that the m6A score was a promising diagnostic biomarker for metastatic prostate cancer.

Lastly, we tested the genetic and epigenetic alterations of all m6A regulators in prostate cancer because these alterations was considered as the common events in other types of cancers. Within 331 prostate cancer samples that underwent whole-genome sequencing, only 19 (5.74\%) patients had genetic variation, and $\mathrm{ZC} 3 \mathrm{H} 13$ was the most common alteration of all m6A regulators. Half m6A regulators (WTAP, ALKBH5, YTHDC1, YTHDF1, YTHDF2, YTHDF3, RNPA2B1, HNPRNPC, FMR1, ELAVL1) did not have any genetic alteration (Supplementary figure 7). We then explored whether the DNA methylation 
profiles of these m6A regulators had changed the expression of m6A regulators. We selected the CpG loci located in the promoter areas of the m6A regulators to compare their methylation levels. Similar to the genetic profiles, the DNA methylation levels were consistent across all prostate cancer in m6A regulators (Supplementary figure 8). Taken together, these results suggested that m6A regulators were stable in prostate cancer.

\section{Discussion}

Prostate cancer remains the most prevalent non-cutaneous carcinoma in men in North America and the second most common cause of cancer death worldwide. The growth of normal and malignant prostate tissue is regulated by androgens through action of the AR in both epithelial and stromal cells. Thus, the primary treatment for metastatic prostate cancer is ADT, and in the majority of patients, this treatment provides a temporary control of the disease. However, cancer cells eventually become castration-resistant, resulting in disease progression to $\mathrm{MCRPC}$. The survival rates for both patients with metastatic prostate cancer at diagnosis and patients with MCRPC upon ADT failure are poor. A few studies uncovering the heterogeneity of mCRPC beyond the somatic mutation highlights the alteration of RNA and transcriptomes. In this study, we demonstrated the subtypes of prostate cancer according to the clustering of $\mathrm{m} 6 \mathrm{~A}$ regulators of expression are significantly associated with prostate cancer metastasis. $\mathrm{M} 6 \mathrm{~A}$ regulators likely contribute to the heterogeneity of prostate cancer metastasis through the key genes involved in immune cell infiltration and metabolism.

Unlike other types of cancer, m6A regulators are not associated with patients' survival or recurrence in primary PCa. We performed a survival analysis of PCa to explore the correlation of survival with m6A subtypes. Surprisingly, there is no statistical significance between m6A subtypes on overall survival or recurrence free survival. That is likely because most primary PCa are biologically slow-growing and the regulatory effects by $\mathrm{m} 6 \mathrm{~A}$ regulators are marginal for primary $\mathrm{PCa}$. Considering the DEGs of $\mathrm{m} 6 \mathrm{~A}$ subtypes are enriched in the metabolic pathway that is important to PCa metastasis, we then investigate the correlation of $\mathrm{m} 6 \mathrm{~A}$ regulator with metastasis.

Metastasis causes more than $90 \%$ of cancer-related deaths, and most prostate cancer patients also die from metastasis (23). The 'metastatic cascade' is a complex biological process that encompasses tumour cell dissociation (from the primary tumour), local invasion, intravasation, transport in circulation, extravasation, colonization, and overt growth in end organs. It has become clear that the development of metastasis not only involves many tumour cell-intrinsic properties but also depends on productive interactions between cancer cells and the tumour microenvironment.

Recent studies also provide evidence that prostate cancer, in the context of ADT-associated metastasis, displays dynamic patterns of evolution (24). Metastasis-to-metastasis spread was found to be common via two mechanisms. First, subclones within a metastasis can originate from another metastatic site rather than the primary tumour in a process called 'cross-metastatic seeding' (25). This phenomenon was also demonstrated in response to therapy in a patient with lethal prostate cancer (26). Second, the same 
sets of subclones can seed multiple sites of metastasis in a process called 'polyclonal seeding' (25). Multiple subclones may be shared between such polyclonal seeds for two or more metastases, suggesting that these subclones might functionally cooperate with one another to promote metastatic progression. Distant metastases could also reseed the surgical bed, suggesting that prostate cancer cells may take advantage of pre-existing supportive niches (26). This process of 'tumour self-seeding' has previously been observed with prostate cancer CTCs, which could lead to the additional recruitment of CTCs and can confer enhanced tumour growth (27).

Prostate tumours are clinically defined as either indolent or aggressive. The majority of these tumours are localized and treated according to their stage or Gleason score, which is determined primarily by biopsy sampling. Most tumours are identified as aggressive or advanced at the time of first diagnosis and are often accompanied by micrometastasis at secondary locations. Of the different sites in the body, the skeletal system has especially high propensity to develop metastatic lesions in patients with prostate cancer (28) (29). The presence of bone metastasis in PCa patients, in addition to visceral metastasis, is associated with a significantly lower overall survival of 14 months (30), signifying the importance of metastatic locations in determining disease prognosis.

A few attempts have been made to predict the metastatic PCa from non-metastatic primary PCa but there is no reliable biomarker yet (31-33). Our m6A score can differentiate the metastatic PCa from nonmetastatic $\mathrm{PCa}$, highlighting its clinical utility though a large scale clinical validation cohort is still required to compared with other biomarkers such as AR-V7 (34). Further investigation on the association of $\mathrm{m} 6 \mathrm{~A}$ regulators with the multiple metabolic pathways in the development of $\mathrm{PCa}$ metastasis is also needed (35).

As mediators of post-transcriptional modification, m6A regulators participate in a variety of biological processes, including cancer tumorigenesis and progression. METTL3 is one of the well-characterized m6A regulators in prostate cancer. Cai et al first reported that METTL3 promoted cell growth in prostate cancer by modifying mRNA of GLI1, the key nuclear mediator in the Hedgehog pathway (36). High expression of METTL3 is also associated with bone metastasis in prostate cancer. By transferring the methyl group to mRNA of ITGB1, METTL3 enhance the prostate cancer cell motility to accommodate bone metastasis (37). In addition, prostate cancer progression is significantly associated with METTL3, suggesting that METTL3 is likely a key regulator in prostate cancer.

Although we established a comprehensive landscape of m6A patterns in prostate cancer and validated our m6A score externally, there are some limitations that need to be addressed. First, the m6A score we constructed should be tested in more independent cohorts to verify its clinical utility. Second, the m6A score is a surrogate for the comprehensive activation of m6A regulators, but the most critical molecule(s) in RNA modification has not been fully investigated in prostate cancer. The detailed mechanism of m6A regulators and the relationship with their targets genes have not yet been elucidated. In addition, the regulatory mechanism of m6A regulators and IncRNA expression has remained unclear (38). Last, the 
driving force of the differential expression pattern of m6A regulators beyond DNA methylation and genomic alteration should also be further investigated in metastatic prostate cancer.

\section{Conclusion}

In conclusion, the clustering of m6A regulators in prostate cancer suggests the heterogeneity of prostate cancer, and one subtype is significantly associated with prostate cancer metastasis. The gene signatures in this "metastatic" prostate cancer are enriched in immune cell response and metabolic response. The m6A score we constructed is potentially a biomarker for metastatic prostate cancer.

\section{Declarations}

\section{Ethics approval and consent to participate}

Not applicable

Consent for publication

All authors agree for publication

\section{Availability of data and materials}

The $\mathrm{R}$ code for this study is released at https://github.com/mackaay/PCa_m6A.

\section{Competing interests}

There is no conflict of interest between authors.

\section{Funding}

The study is supported by National Natural Science Foundation of China (NSFC) $(81803900,81802569)$

\section{Authors' contributions}

Study design: XW, CM, HJ, JH

Bioinformatic analysis: XW, QG, CY, CM

Results interpretation: $\mathrm{XW}, \mathrm{CY}, \mathrm{YW}, \mathrm{MH}, \mathrm{HJ}$

Manuscript writing: $\mathrm{XW}, \mathrm{CM}, \mathrm{HJ}, \mathrm{JH}$

\section{Acknowledgements}

Not applicable 


\section{Code availability}

The R code for this study is released at https://github.com/mackaay/PCa_m6A.

\section{References}

1. Liu D, Kuai Y, Zhu R, Zhou C, Tao Y, Han W, et al. Prognosis of prostate cancer and bone metastasis pattern of patients: a SEER-based study and a local hospital based study from China. Sci Rep 2020;10:9104

2. Welch HG, Albertsen PC. Reconsidering Prostate Cancer Mortality - The Future of PSA Screening. N Engl J Med 2020;382:1557-63

3. Perlmutter MA, Lepor H. Androgen deprivation therapy in the treatment of advanced prostate cancer. Rev Urol 2007;9 Suppl 1:S3-8

4. Corn PG, Heath El, Zurita A, Ramesh N, Xiao L, Sei E, et al. Cabazitaxel plus carboplatin for the treatment of men with metastatic castration-resistant prostate cancers: a randomised, open-label, phase 1-2 trial. Lancet Oncol 2019;20:1432-43

5. Khalaf DJ, Annala M, Taavitsainen S, Finch DL, Oja C, Vergidis J, et al. Optimal sequencing of enzalutamide and abiraterone acetate plus prednisone in metastatic castration-resistant prostate cancer: a multicentre, randomised, open-label, phase 2, crossover trial. Lancet Oncol 2019;20:1730-9

6. Mateo J, Porta N, Bianchini D, McGovern U, Elliott T, Jones R, et al. Olaparib in patients with metastatic castration-resistant prostate cancer with DNA repair gene aberrations (TOPARP-B): a multicentre, open-label, randomised, phase 2 trial. Lancet Oncol 2020;21:162-74

7. de Bono J, Mateo J, Fizazi K, Saad F, Shore N, Sandhu S, et al. Olaparib for Metastatic CastrationResistant Prostate Cancer. N Engl J Med 2020;382:2091-102

8. Zhang Z, Zhou C, Li X, Barnes SD, Deng S, Hoover E, et al. Loss of CHD1 Promotes Heterogeneous Mechanisms of Resistance to AR-Targeted Therapy via Chromatin Dysregulation. Cancer Cell 2020;37:584-98 e11

9. Watson PA, Arora VK, Sawyers CL. Emerging mechanisms of resistance to androgen receptor inhibitors in prostate cancer. Nat Rev Cancer 2015;15:701-11

10. Zhang Z, Luo K, Zou Z, Qiu M, Tian J, Sieh L, et al. Genetic analyses support the contribution of mRNA N(6)-methyladenosine (m(6)A) modification to human disease heritability. Nat Genet 2020

11. Zaccara S, Ries RJ, Jaffrey SR. Reading, writing and erasing mRNA methylation. Nat Rev Mol Cell Biol 2019;20:608-24

12. Yoon KJ, Ringeling FR, Vissers $C$, Jacob F, Pokrass M, Jimenez-Cyrus D, et al. Temporal Control of Mammalian Cortical Neurogenesis by m(6)A Methylation. Cell 2017;171:877-89 e17

13. Shi H, Zhang X, Weng YL, Lu Z, Liu Y, Lu Z, et al. m(6)A facilitates hippocampus-dependent learning and memory through YTHDF1. Nature 2018;563:249-53 
14. Bertero A, Brown S, Madrigal P, Osnato A, Ortmann D, Yiangou L, et al. The SMAD2/3 interactome reveals that TGFbeta controls m(6)A mRNA methylation in pluripotency. Nature 2018;555:256-9

15. Geng Y, Guan R, Hong W, Huang B, Liu P, Guo X, et al. Identification of m6A-related genes and m6A RNA methylation regulators in pancreatic cancer and their association with survival. Ann Transl Med 2020;8:387

16. Chen X, Xu M, Xu X, Zeng K, Liu X, Pan B, et al. METTL14-mediated N6-methyladenosine modification of SOX4 mRNA inhibits tumor metastasis in colorectal cancer. Mol Cancer 2020;19:106

17. Zhang C, Huang S, Zhuang H, Ruan S, Zhou Z, Huang K, et al. YTHDF2 promotes the liver cancer stem cell phenotype and cancer metastasis by regulating OCT4 expression via m6A RNA methylation. Oncogene 2020;39:4507-18

18. Zheng Z, Mao S, Guo Y, Zhang W, Liu J, Li C, et al. N6methyladenosine RNA methylation regulators participate in malignant progression and have prognostic value in clear cell renal cell carcinoma. Oncol Rep 2020;43:1591-605

19. Zhu J, Wang M, Hu D. Deciphering N(6)-Methyladenosine-Related Genes Signature to Predict Survival in Lung Adenocarcinoma. Biomed Res Int 2020;2020:2514230

20. Malta TM, Sokolov A, Gentles AJ, Burzykowski T, Poisson L, Weinstein JN, et al. Machine Learning Identifies Stemness Features Associated with Oncogenic Dedifferentiation. Cell 2018;173:338-54 e15

21. Lu X, Horner JW, Paul E, Shang X, Troncoso P, Deng P, et al. Effective combinatorial immunotherapy for castration-resistant prostate cancer. Nature 2017;543:728-32

22. Shen C, Sheng Y, Zhu AC, Robinson S, Jiang X, Dong L, et al. RNA Demethylase ALKBH5 Selectively Promotes Tumorigenesis and Cancer Stem Cell Self-Renewal in Acute Myeloid Leukemia. Cell Stem Cell 2020;27:64-80 e9

23. Rycaj K, Li H, Zhou J, Chen X, Tang DG. Cellular determinants and microenvironmental regulation of prostate cancer metastasis. Semin Cancer Biol 2017;44:83-97

24. Shen MM. Cancer: The complex seeds of metastasis. Nature 2015;520:298-9

25. Gundem G, Van Loo P, Kremeyer B, Alexandrov LB, Tubio JMC, Papaemmanuil E, et al. The evolutionary history of lethal metastatic prostate cancer. Nature 2015;520:353-7

26. Hong MK, Macintyre G, Wedge DC, Van Loo P, Patel K, Lunke S, et al. Tracking the origins and drivers of subclonal metastatic expansion in prostate cancer. Nat Commun 2015;6:6605

27. Kim MY, Oskarsson T, Acharyya S, Nguyen DX, Zhang XH, Norton L, et al. Tumor self-seeding by circulating cancer cells. Cell 2009;139:1315-26

28. Chambers AF, Groom AC, MacDonald IC. Dissemination and growth of cancer cells in metastatic sites. Nat Rev Cancer 2002;2:563-72

29. Msaouel P, Pissimissis N, Halapas A, Koutsilieris M. Mechanisms of bone metastasis in prostate cancer: clinical implications. Best Pract Res Clin Endocrinol Metab 2008;22:341-55

30. Gandaglia G, Karakiewicz PI, Briganti A, Passoni NM, Schiffmann J, Trudeau V, et al. Impact of the Site of Metastases on Survival in Patients with Metastatic Prostate Cancer. Eur Urol 2015;68:325-34 
31. Kretschmer A, Tilki D. Biomarkers in prostate cancer - Current clinical utility and future perspectives. Crit Rev Oncol Hematol 2017;120:180-93

32. Yang L, Roberts D, Takhar M, Erho N, Bibby BAS, Thiruthaneeswaran N, et al. Development and Validation of a 28-gene Hypoxia-related Prognostic Signature for Localized Prostate Cancer. EBioMedicine 2018;31:182-9

33. Locke WJ, Guanzon D, Ma C, Liew YJ, Duesing KR, Fung KYC, et al. DNA Methylation Cancer Biomarkers: Translation to the Clinic. Front Genet 2019;10:1150

34. Bastos DA, Antonarakis ES. CTC-derived AR-V7 detection as a prognostic and predictive biomarker in advanced prostate cancer. Expert Rev Mol Diagn 2018;18:155-63

35. Torrano V, Valcarcel-Jimenez L, Cortazar AR, Liu X, Urosevic J, Castillo-Martin M, et al. The metabolic co-regulator PGC1 alpha suppresses prostate cancer metastasis. Nat Cell Biol 2016;18:645-56

36. Cai J, Yang F, Zhan H, Situ J, Li W, Mao Y, et al. RNA m(6)A Methyltransferase METTL3 Promotes The Growth Of Prostate Cancer By Regulating Hedgehog Pathway. Onco Targets Ther 2019;12:914352

37. Li E, Wei B, Wang X, Kang R. METTL3 enhances cell adhesion through stabilizing integrin beta1 mRNA via an m6A-HuR-dependent mechanism in prostatic carcinoma. Am J Cancer Res 2020;10:1012-25

38. Barros-Silva D, Lobo J, Guimaraes-Teixeira C, Carneiro I, Oliveira J, Martens-Uzunova ES, et al. VIRMADependent N6-Methyladenosine Modifications Regulate the Expression of Long Non-Coding RNAs CCAT1 and CCAT2 in Prostate Cancer. Cancers (Basel) 2020;12

\section{Tables}

Due to technical limitations, table 1 is only available as a download in the Supplemental Files section.

\section{Figures}


A
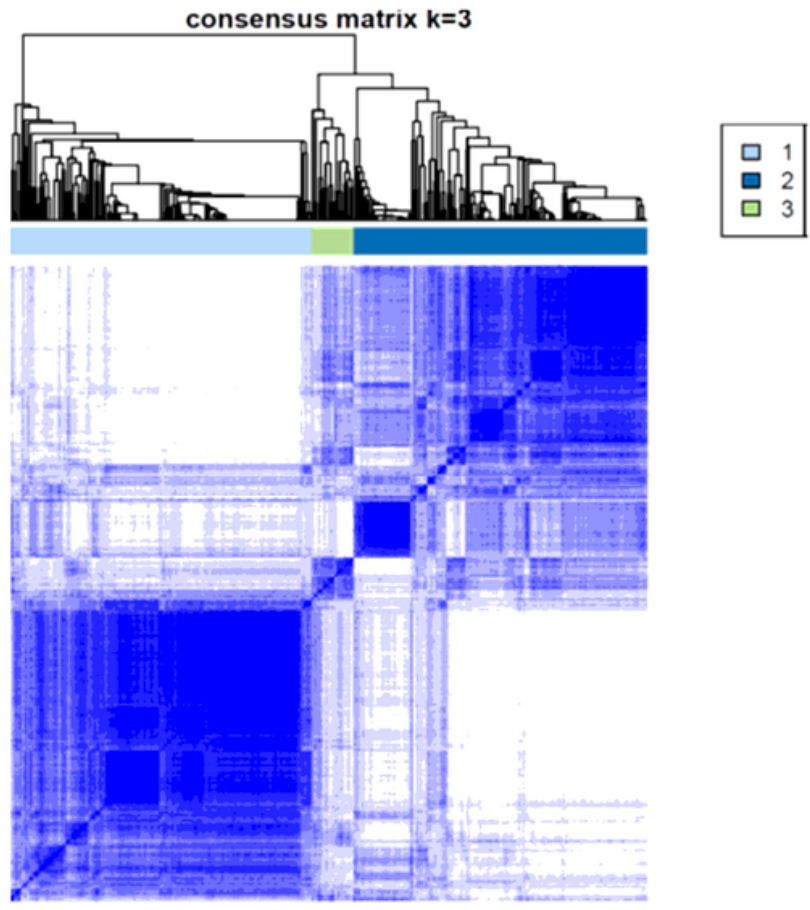

C

\section{Silhouette plot}

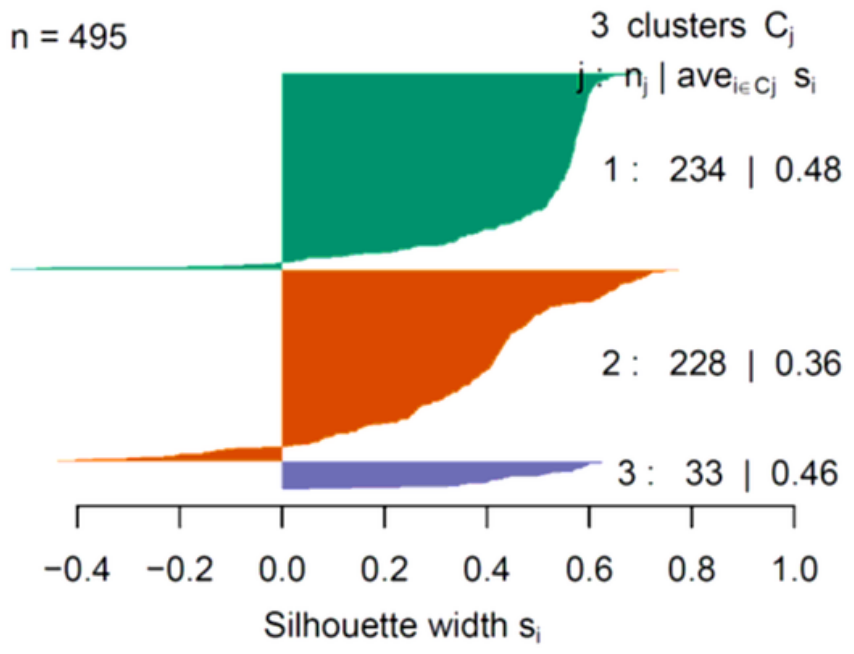

Average silhouette width : 0.42
B

Clustering by $\mathrm{m6A}$ regulators

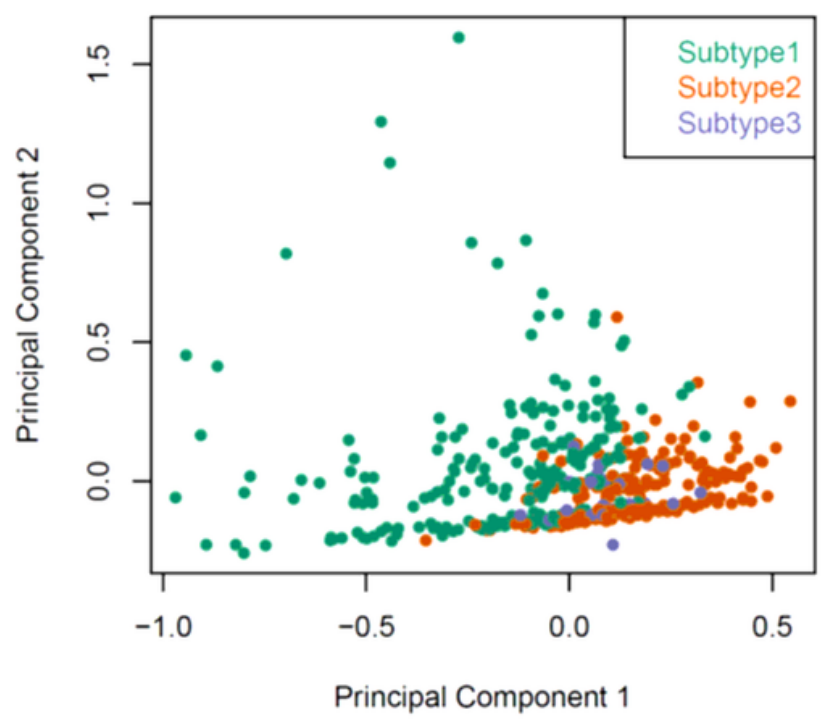

D

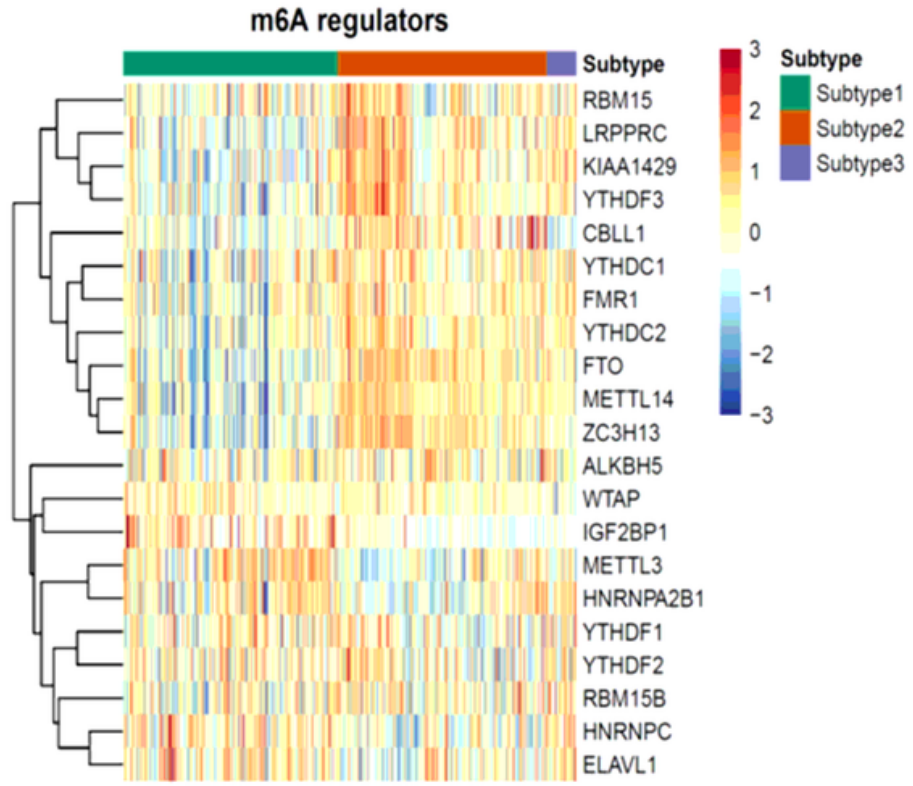

\section{Figure 1}

Consensus clustering of $\mathrm{m} 6 \mathrm{~A}$ regulators demonstrates three distinct subtypes in prostate cancer. $\mathrm{A}$, Heatmap showing the consensus matrix $(k=3)$ of m6A regulator expression. $B, P C A$ showing the clustering of prostate cancer by m6A regulators. C, Silhouette plot showing the similarity of samples clustered in sample subtypes. D, Heatmap showing the distinct pattern of each m6A regulator in all 454 prostate cancer samples. 

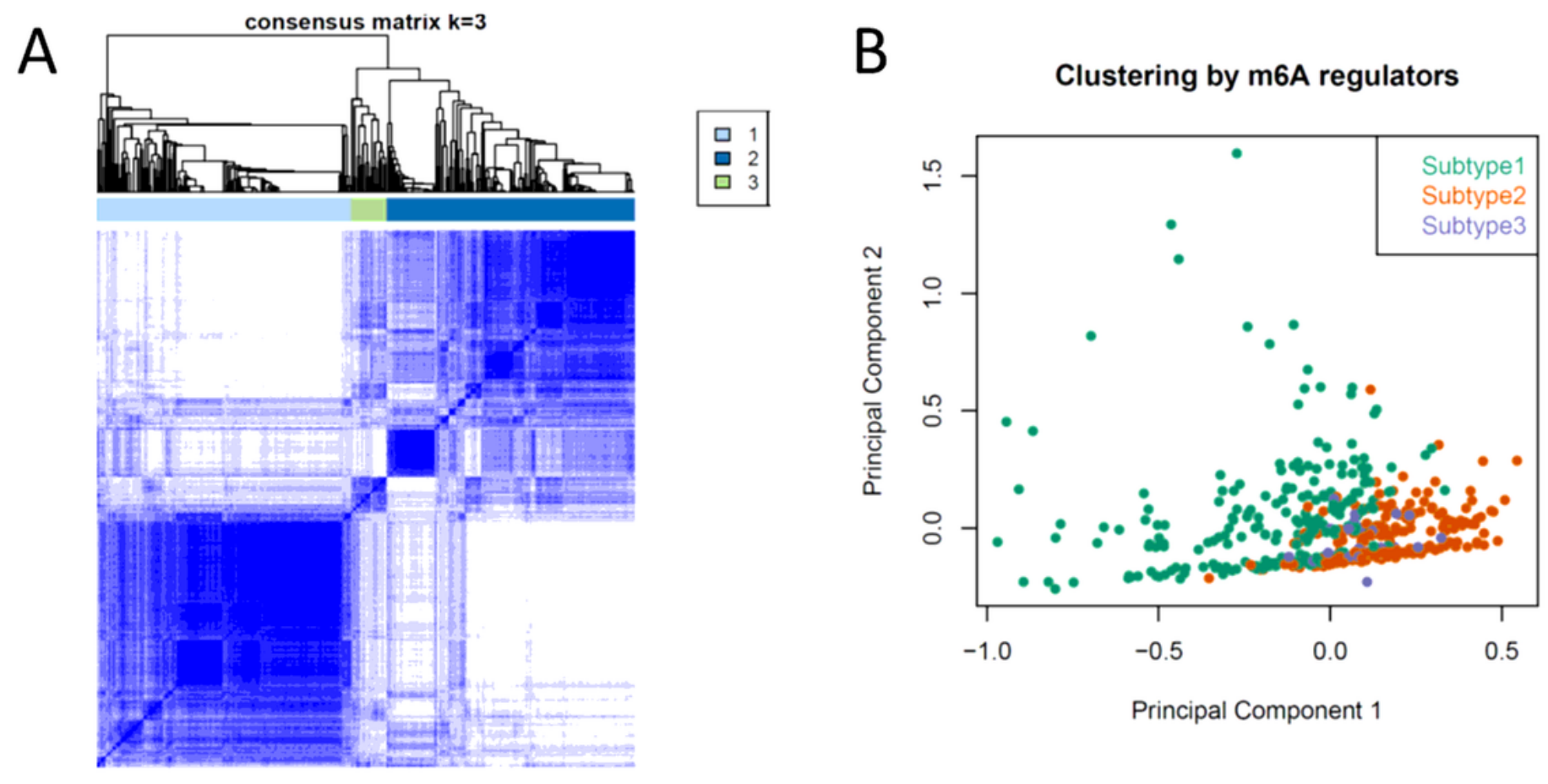

C

\section{Silhouette plot}

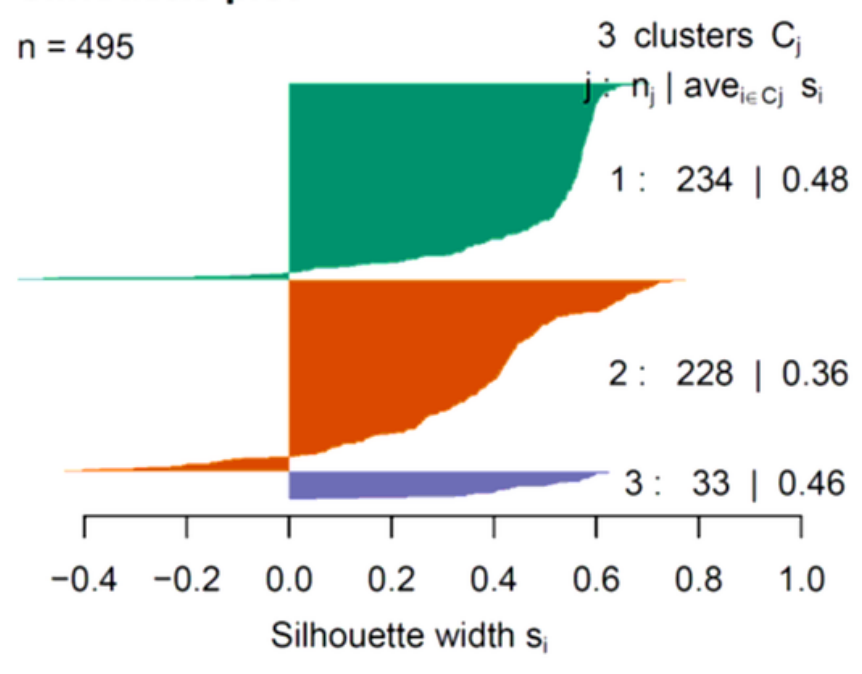

Average silhouette width : 0.42

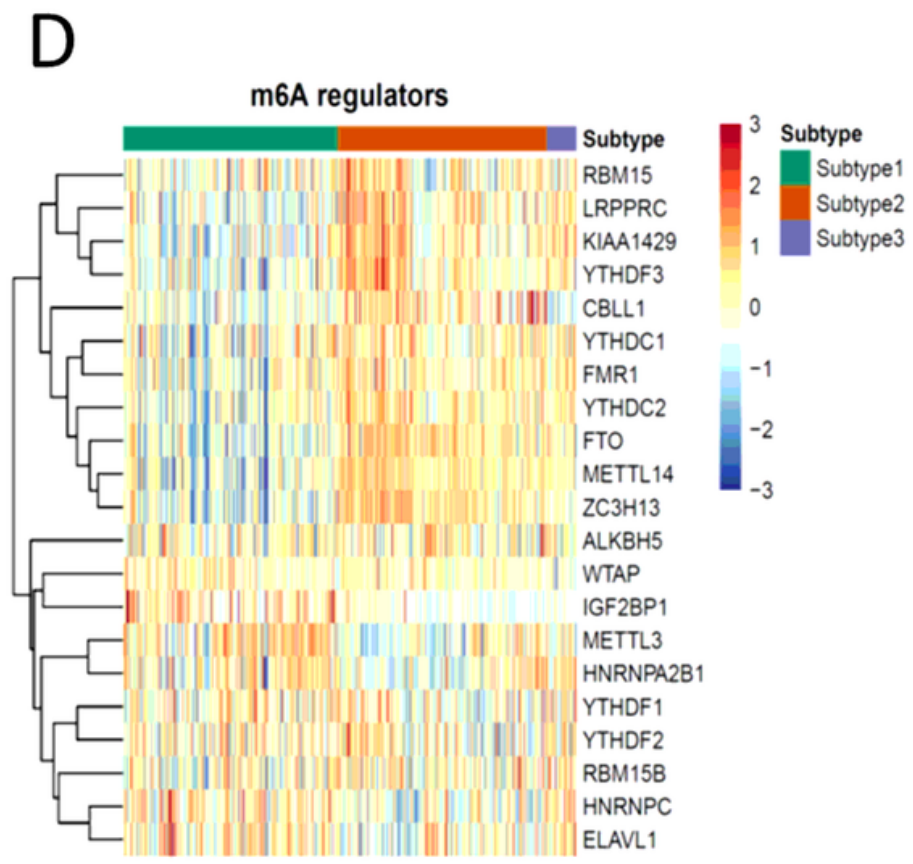

\section{Figure 1}

Consensus clustering of $\mathrm{m} 6 \mathrm{~A}$ regulators demonstrates three distinct subtypes in prostate cancer. $A$, Heatmap showing the consensus matrix $(k=3)$ of m6A regulator expression. B, PCA showing the clustering of prostate cancer by m6A regulators. C, Silhouette plot showing the similarity of samples clustered in sample subtypes. D, Heatmap showing the distinct pattern of each m6A regulator in all 454 prostate cancer samples. 

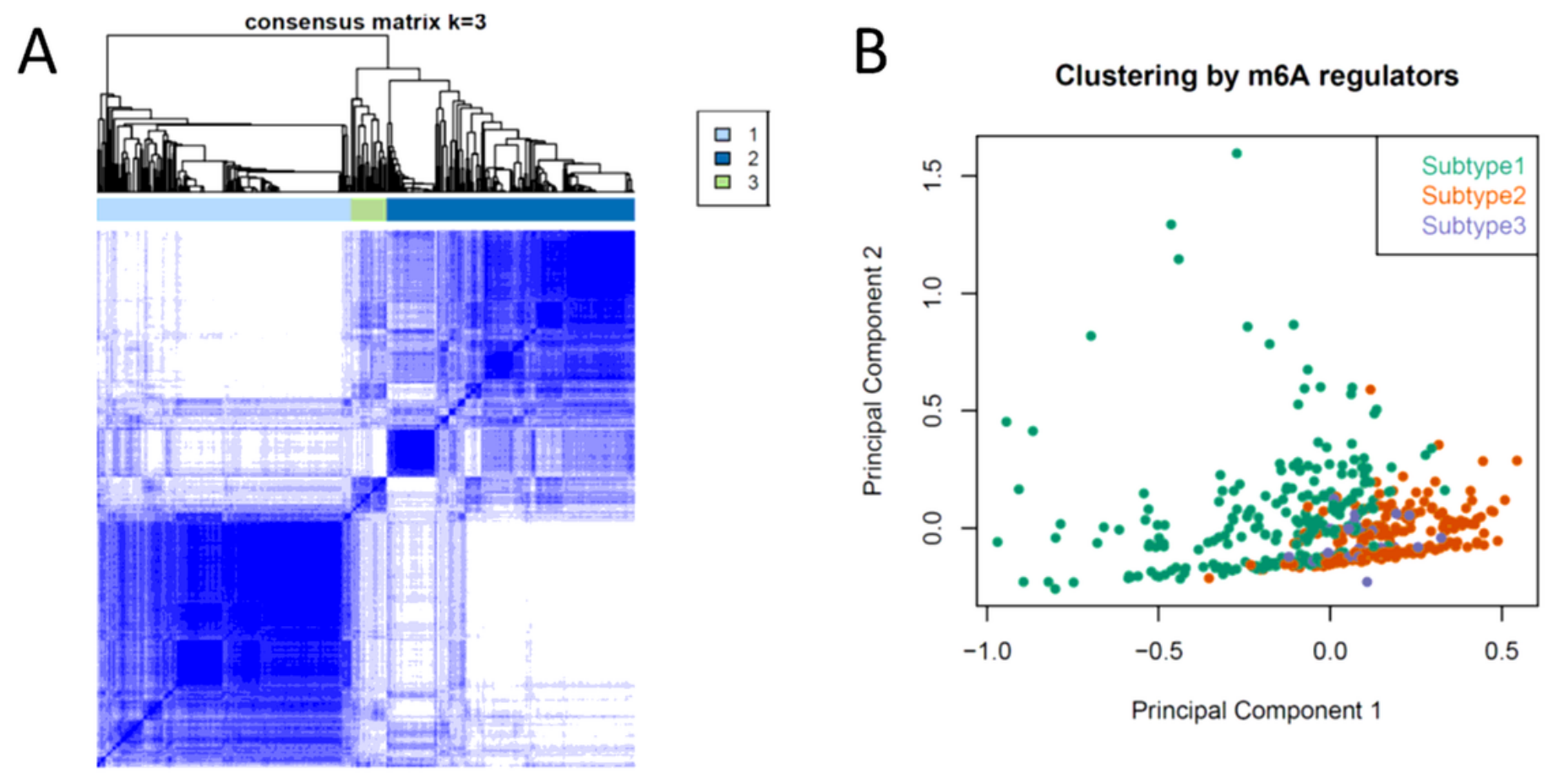

C

\section{Silhouette plot}

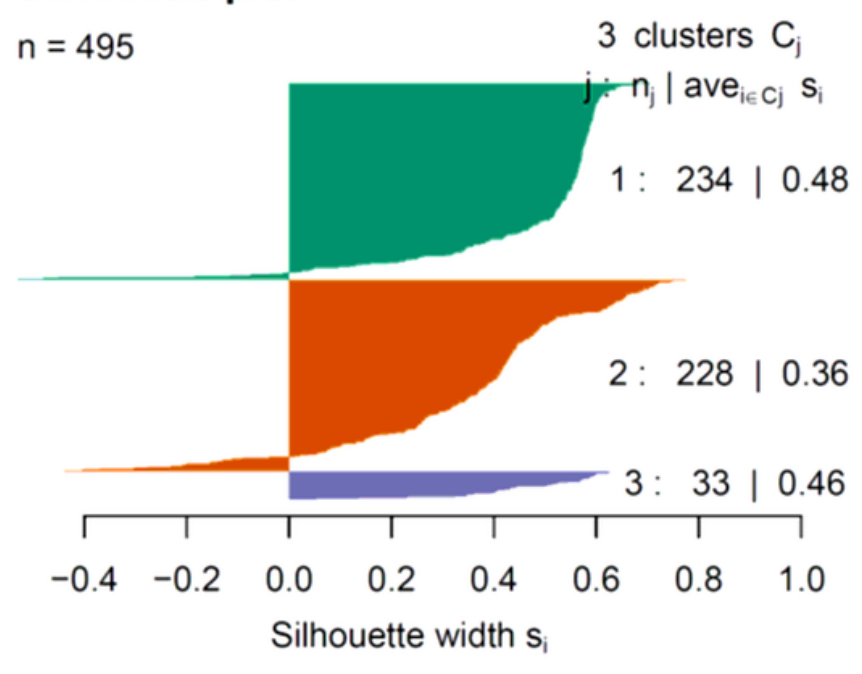

Average silhouette width : 0.42

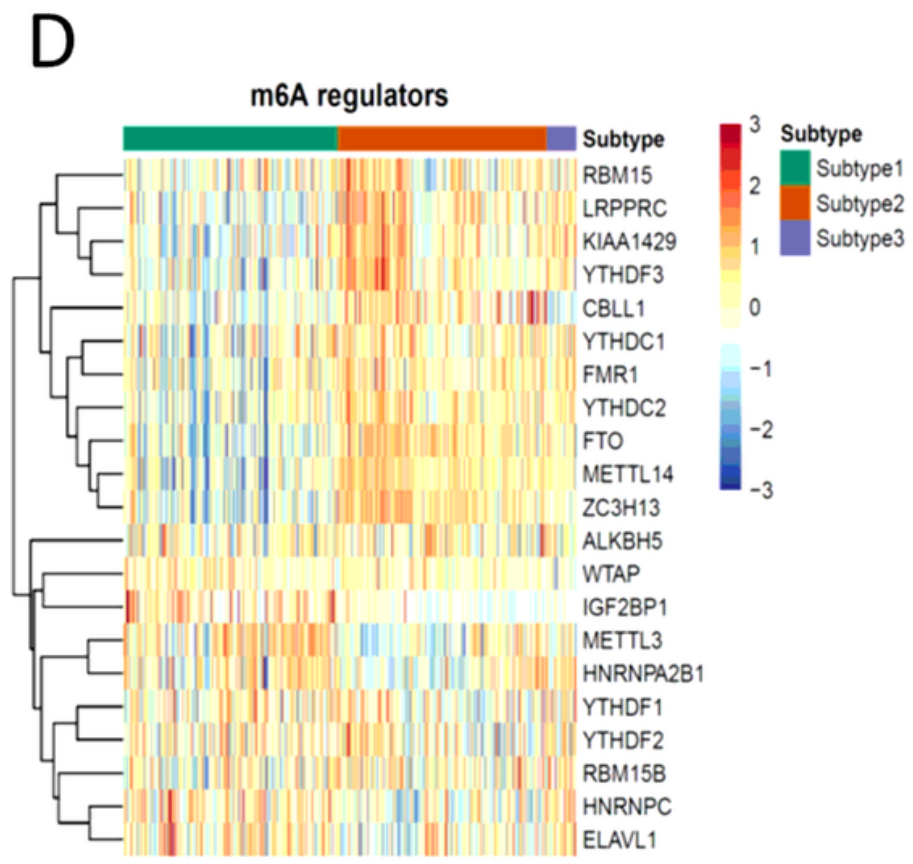

\section{Figure 1}

Consensus clustering of $\mathrm{m} 6 \mathrm{~A}$ regulators demonstrates three distinct subtypes in prostate cancer. $A$, Heatmap showing the consensus matrix $(k=3)$ of m6A regulator expression. B, PCA showing the clustering of prostate cancer by m6A regulators. C, Silhouette plot showing the similarity of samples clustered in sample subtypes. D, Heatmap showing the distinct pattern of each m6A regulator in all 454 prostate cancer samples. 

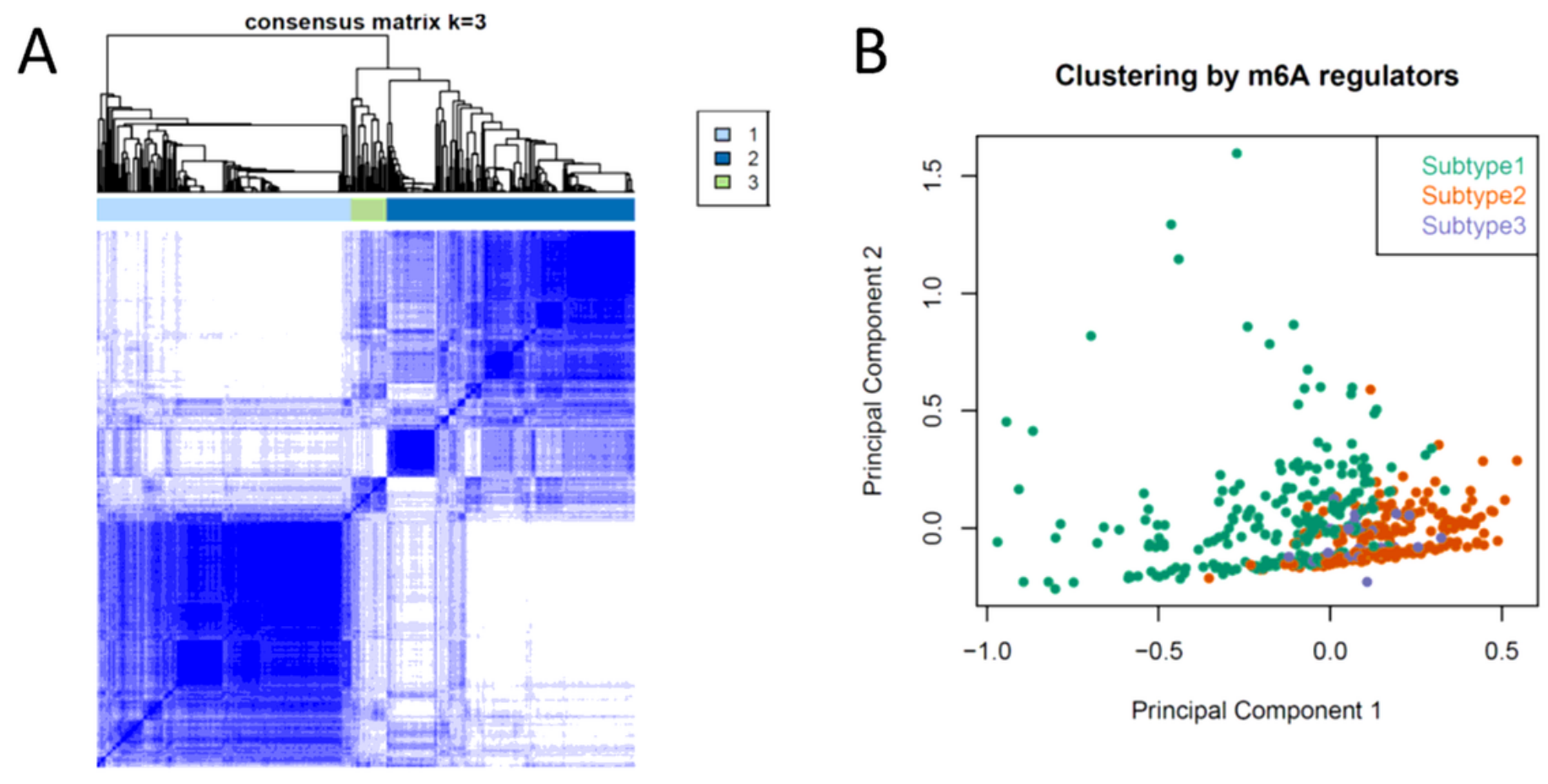

C

\section{Silhouette plot}

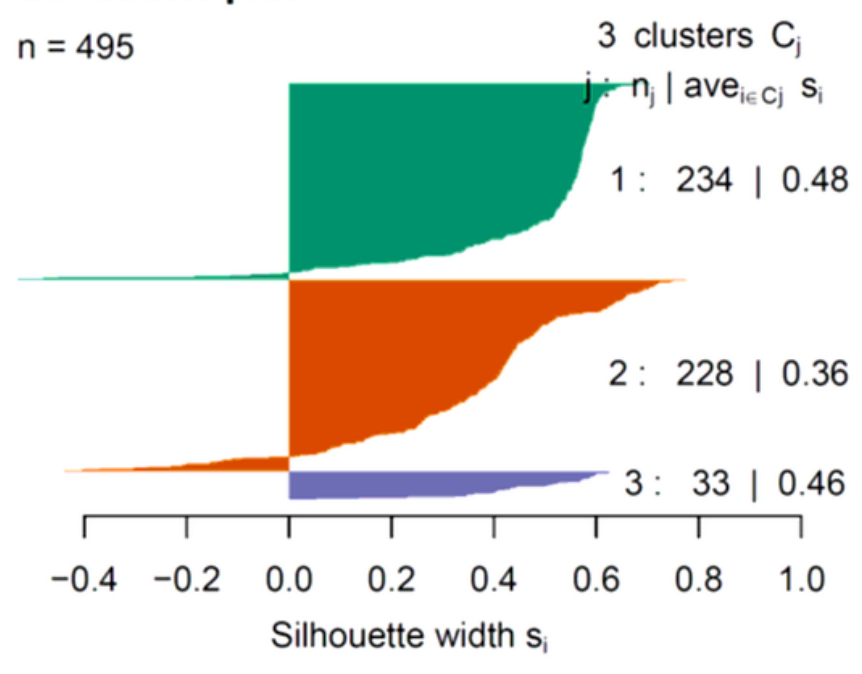

Average silhouette width : 0.42

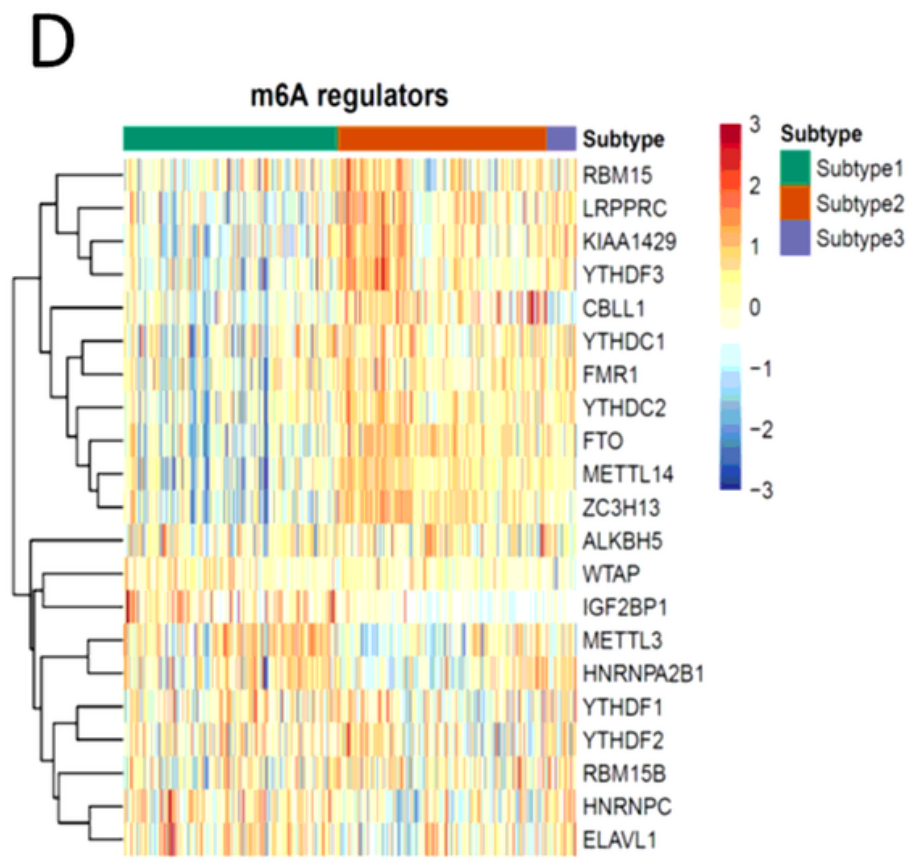

\section{Figure 1}

Consensus clustering of $\mathrm{m} 6 \mathrm{~A}$ regulators demonstrates three distinct subtypes in prostate cancer. $A$, Heatmap showing the consensus matrix $(k=3)$ of m6A regulator expression. B, PCA showing the clustering of prostate cancer by m6A regulators. C, Silhouette plot showing the similarity of samples clustered in sample subtypes. D, Heatmap showing the distinct pattern of each m6A regulator in all 454 prostate cancer samples. 
A
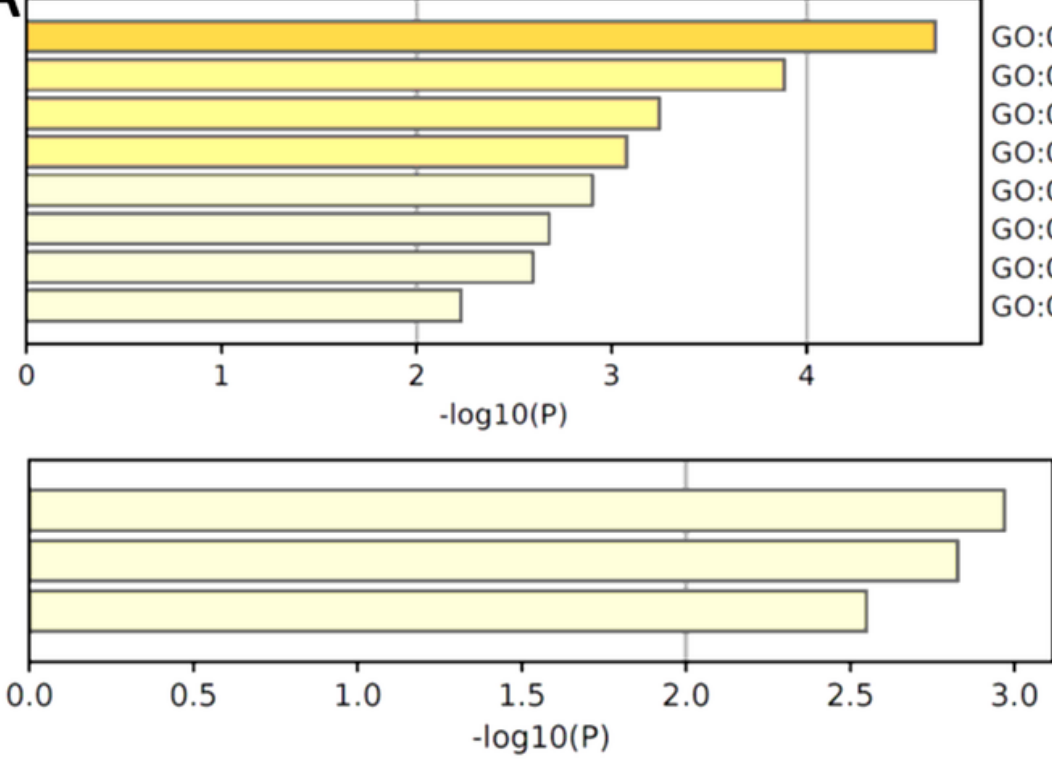

B
GO:0019369: arachidonic acid metabolic process GO:0044264: cellular polysaccharide metabolic process GO:0002819: regulation of adaptive immune response GO:0051047: positive regulation of secretion GO:0048871: multicellular organismal homeostasis GO:0050730: regulation of peptidyl-tyrosine phosphorylation GO:0043270: positive regulation of ion transport GO:0055080: cation homeostasis
Tissue-specific: prostate Tissue-specific: pancreas Tissue-specific: liver

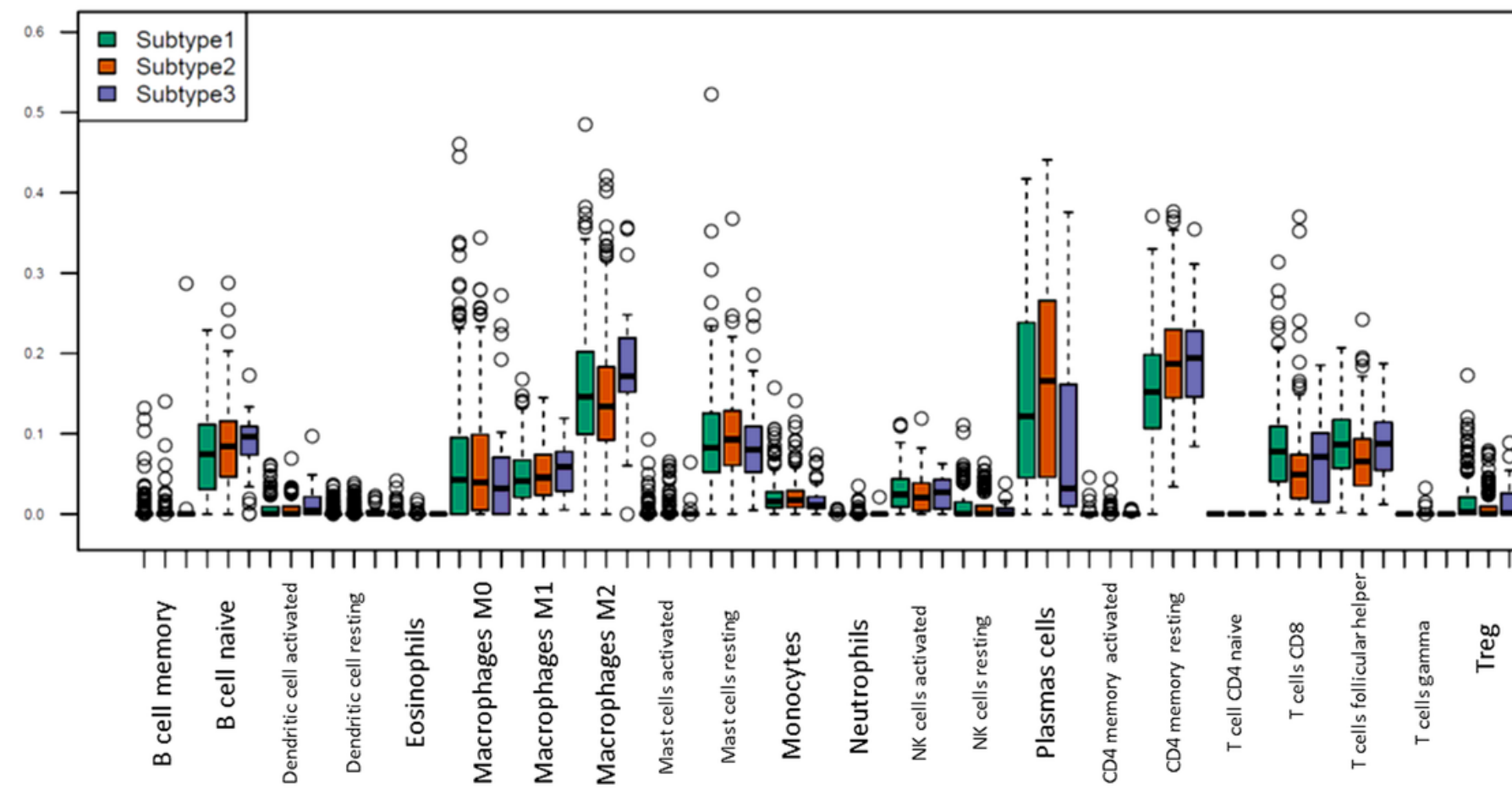

\section{Figure 2}

Different responses of immune cells are enriched in the three subtypes of prostate cancer. A, Pathways of DEG between m6A subtypes are enriched in metabolic process and immune response (upper). The subtype-specific DEGs are enriched in prostate cancer (lower). B, Boxplot showing the different immune cell infiltrations between m6A subtypes. ANOVA test; $* \star \star, p<0.0001$. 
A
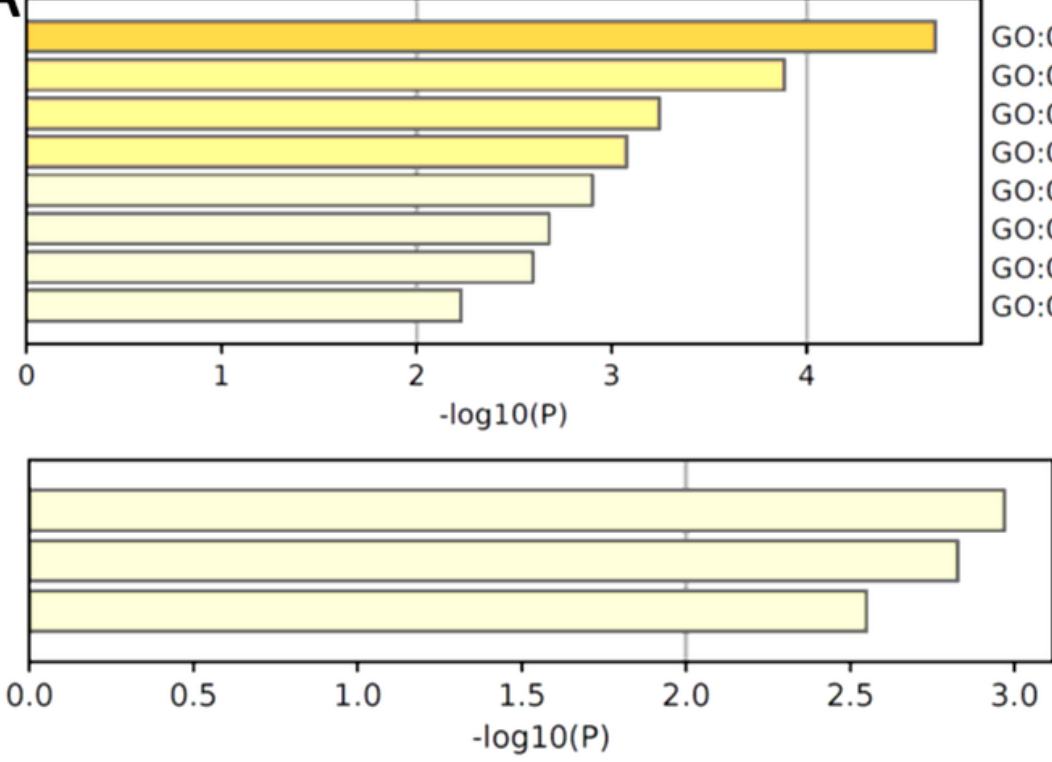

B
GO:0019369: arachidonic acid metabolic process GO:0044264: cellular polysaccharide metabolic process GO:0002819: regulation of adaptive immune response GO:0051047: positive regulation of secretion GO:0048871: multicellular organismal homeostasis GO:0050730: regulation of peptidyl-tyrosine phosphorylation GO:0043270: positive regulation of ion transport GO:0055080: cation homeostasis
Tissue-specific: prostate Tissue-specific: pancreas Tissue-specific: liver

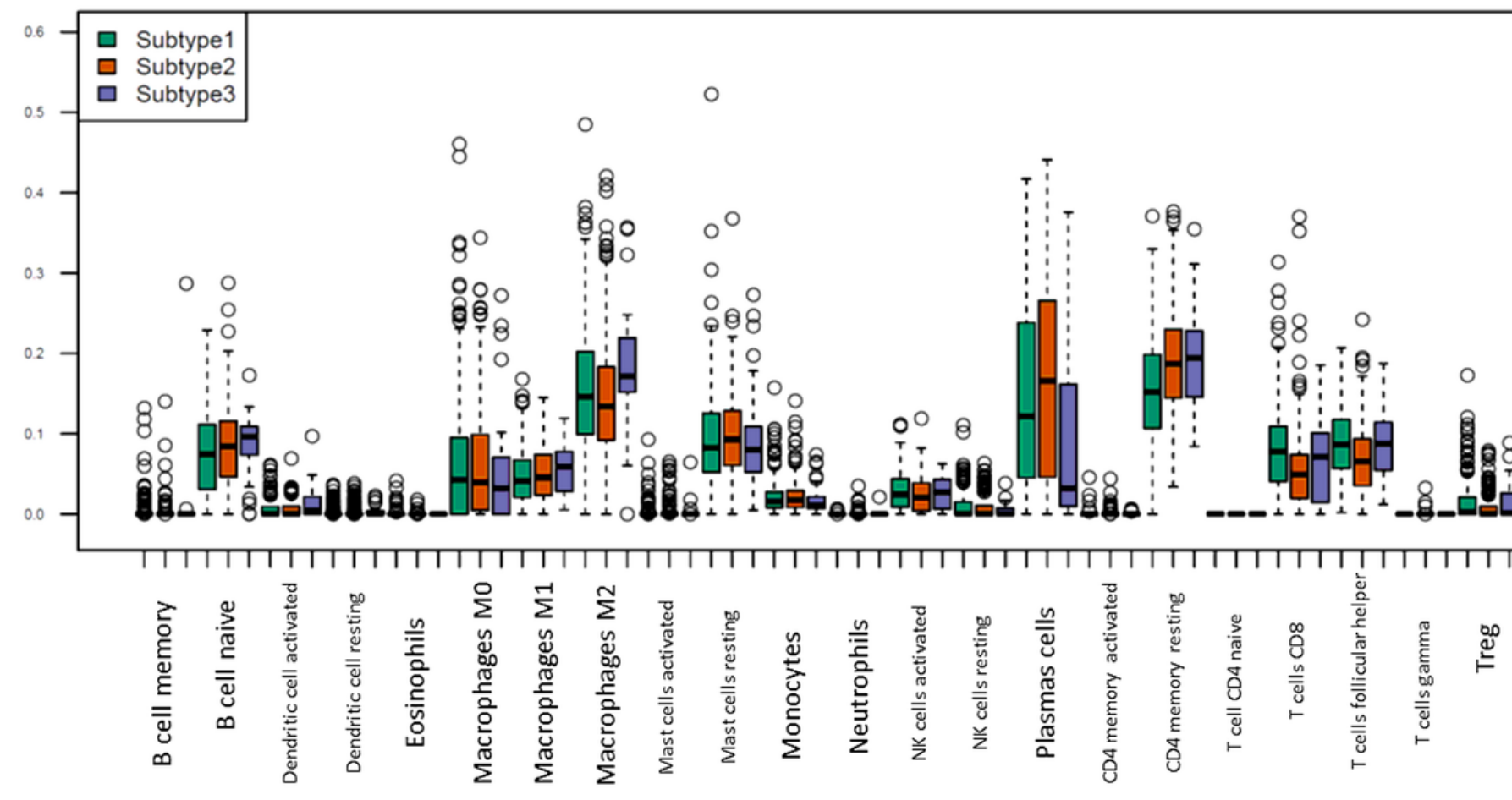

\section{Figure 2}

Different responses of immune cells are enriched in the three subtypes of prostate cancer. A, Pathways of DEG between m6A subtypes are enriched in metabolic process and immune response (upper). The subtype-specific DEGs are enriched in prostate cancer (lower). B, Boxplot showing the different immune cell infiltrations between m6A subtypes. ANOVA test; $* \star \star, p<0.0001$. 
A
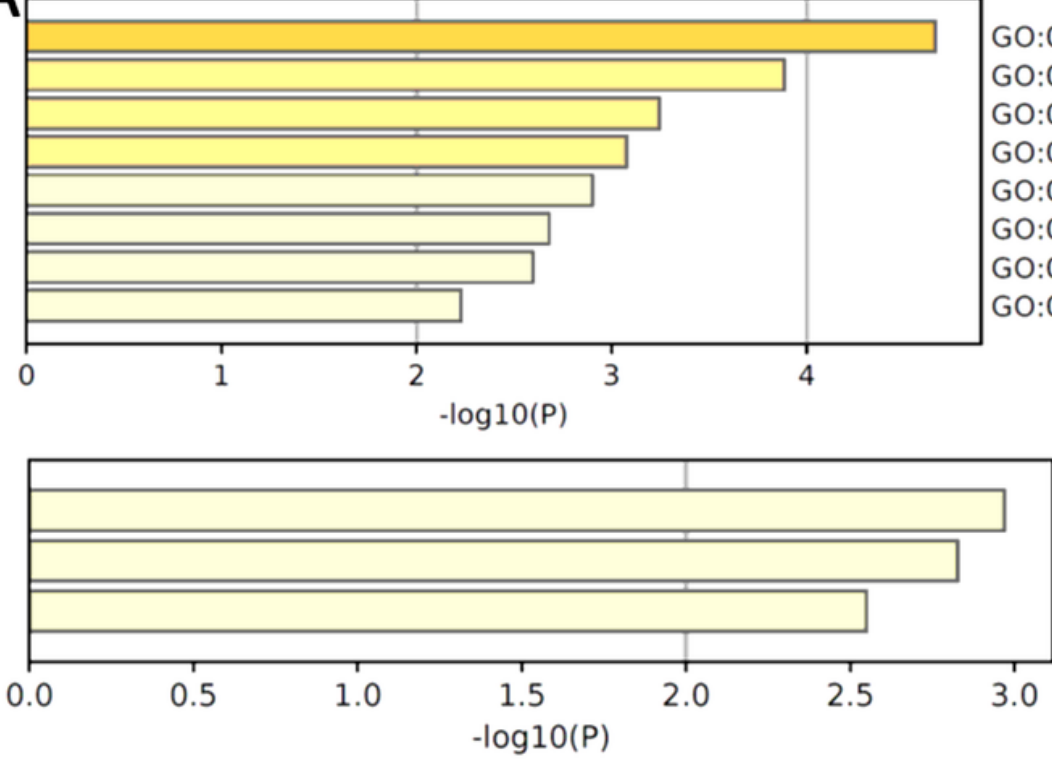

B
GO:0019369: arachidonic acid metabolic process GO:0044264: cellular polysaccharide metabolic process GO:0002819: regulation of adaptive immune response GO:0051047: positive regulation of secretion GO:0048871: multicellular organismal homeostasis GO:0050730: regulation of peptidyl-tyrosine phosphorylation GO:0043270: positive regulation of ion transport GO:0055080: cation homeostasis
Tissue-specific: prostate Tissue-specific: pancreas Tissue-specific: liver

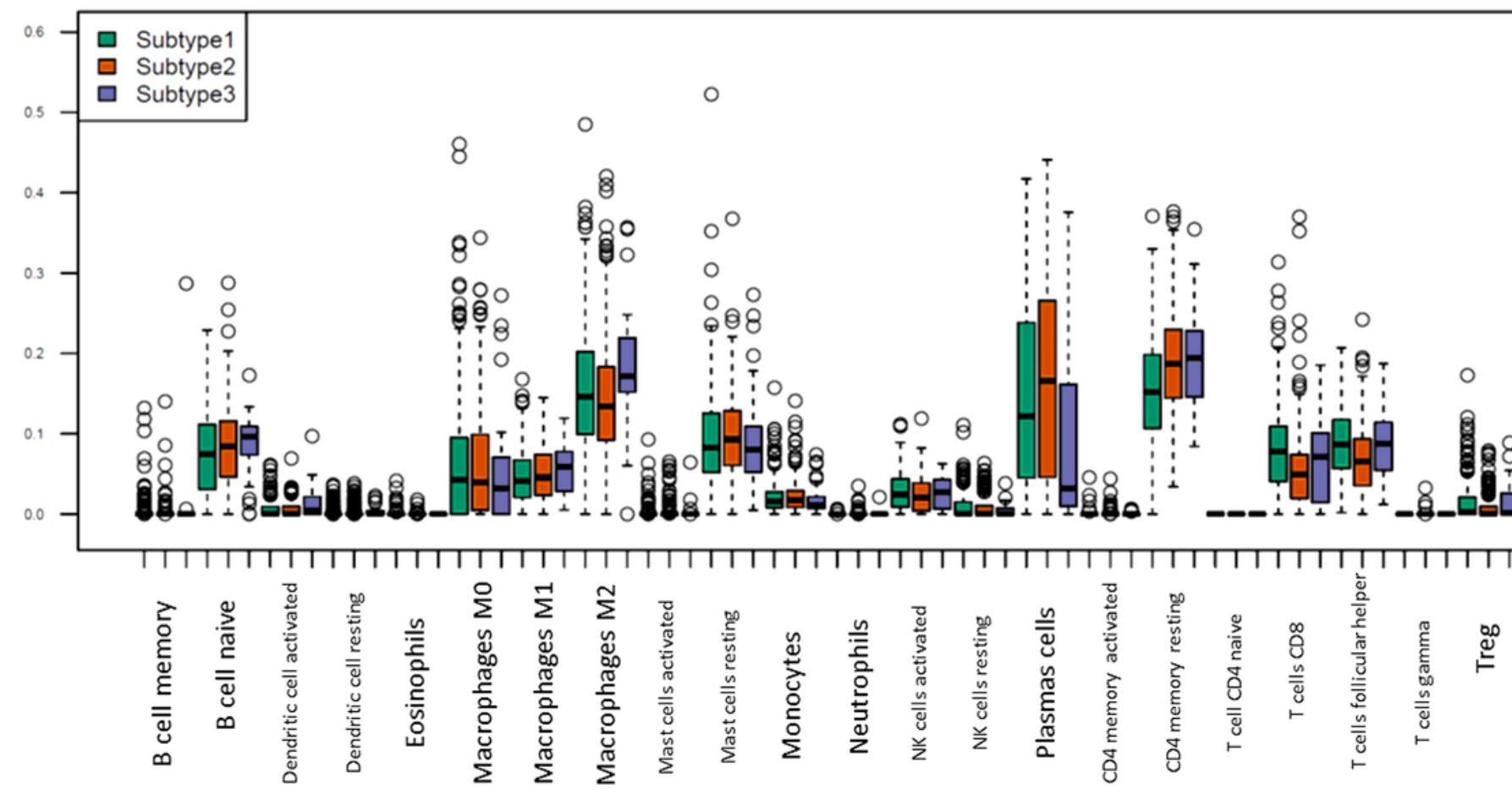

\section{Figure 2}

Different responses of immune cells are enriched in the three subtypes of prostate cancer. A, Pathways of DEG between m6A subtypes are enriched in metabolic process and immune response (upper). The subtype-specific DEGs are enriched in prostate cancer (lower). B, Boxplot showing the different immune cell infiltrations between m6A subtypes. ANOVA test; $* \star \star, p<0.0001$. 
A
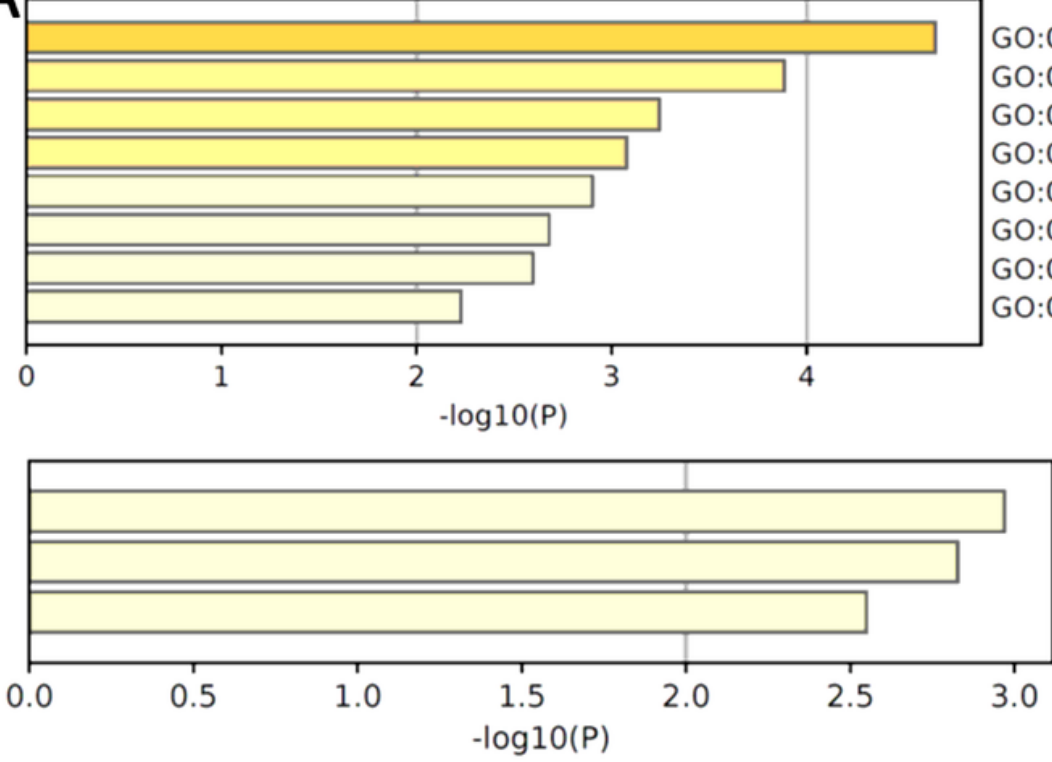

B
GO:0019369: arachidonic acid metabolic process GO:0044264: cellular polysaccharide metabolic process GO:0002819: regulation of adaptive immune response GO:0051047: positive regulation of secretion GO:0048871: multicellular organismal homeostasis GO:0050730: regulation of peptidyl-tyrosine phosphorylation GO:0043270: positive regulation of ion transport GO:0055080: cation homeostasis
Tissue-specific: prostate Tissue-specific: pancreas Tissue-specific: liver

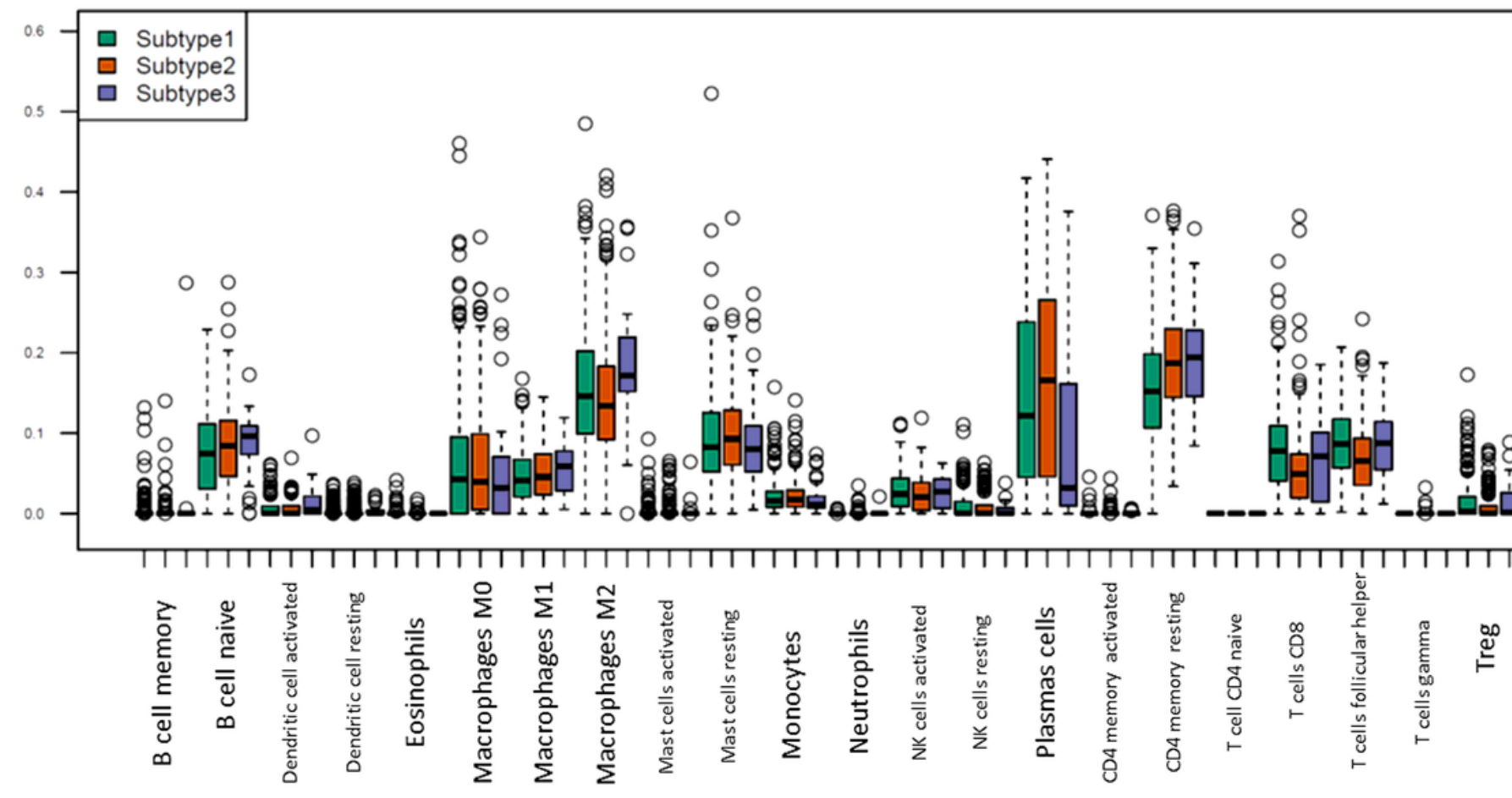

\section{Figure 2}

Different responses of immune cells are enriched in the three subtypes of prostate cancer. A, Pathways of DEG between m6A subtypes are enriched in metabolic process and immune response (upper). The subtype-specific DEGs are enriched in prostate cancer (lower). B, Boxplot showing the different immune cell infiltrations between m6A subtypes. ANOVA test; $* \star \star, p<0.0001$. 


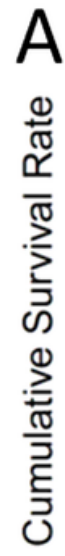

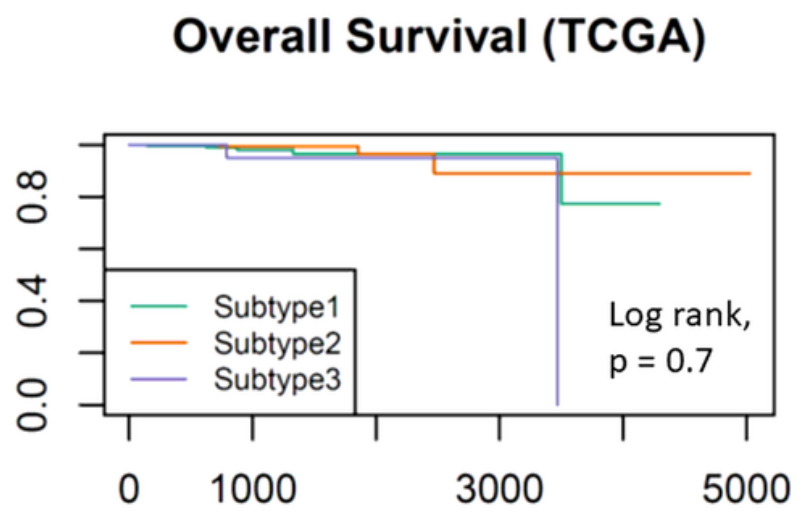

Days

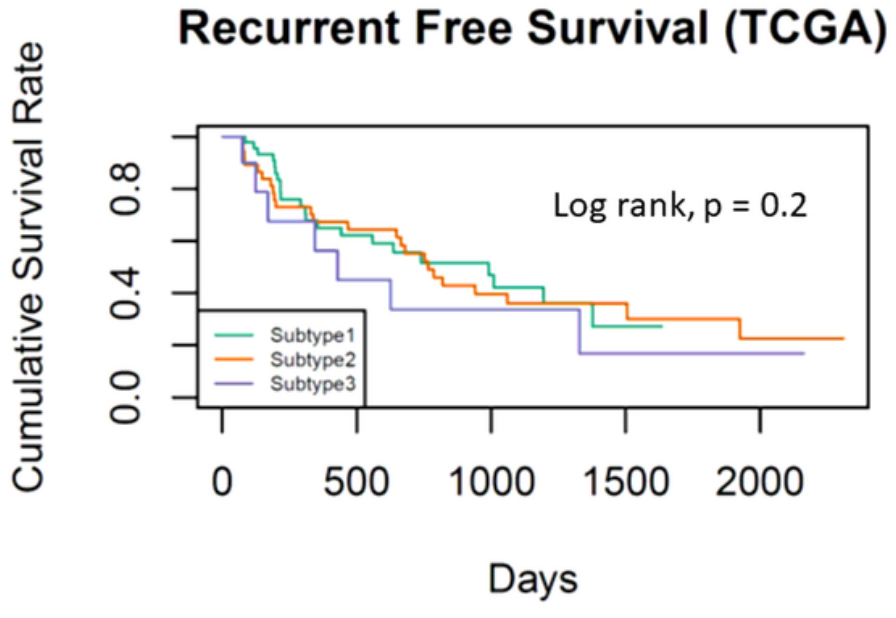

B

m6A Regulators Expression in GSE147493

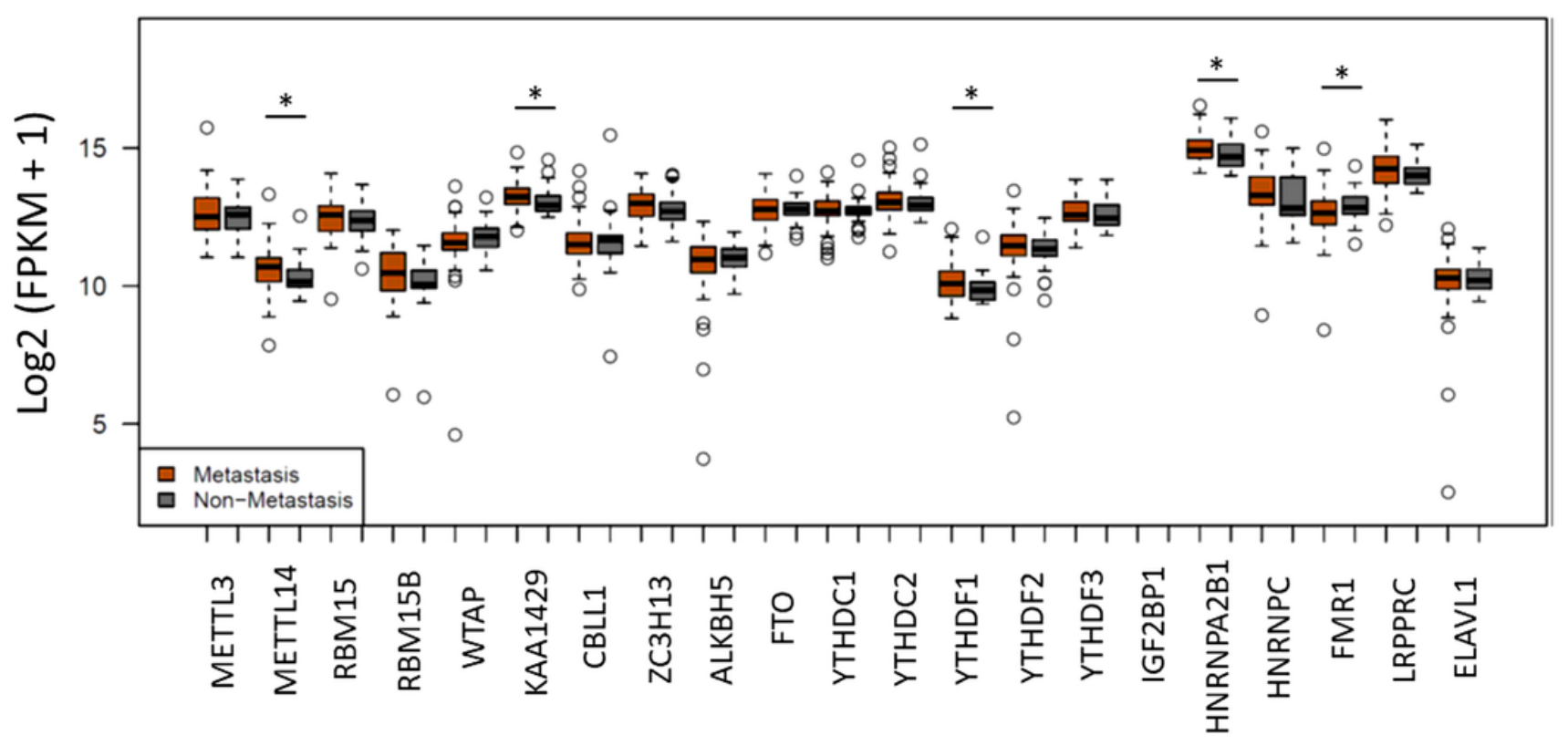

Figure 3

The regulators of $\mathrm{m} 6 \mathrm{~A}$ are more relevant to metastasis than recurrence in prostate cancer. A, Subtyping of prostate cancer by m6A regulators is not associated with patients' overall and recurrent-free survival (Log rank test, $p=0.7$ and 0.2 , respectively). $B, m 6 A$ regulators are associated with prostate cancer metastasis. Seven $\mathrm{m} 6 \mathrm{~A}$ regulators are differentially expressed between metastatic and non-metastatic prostate cancer. Student's t test. *, $\mathrm{p}<0.05$. 


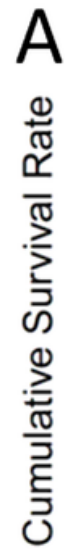

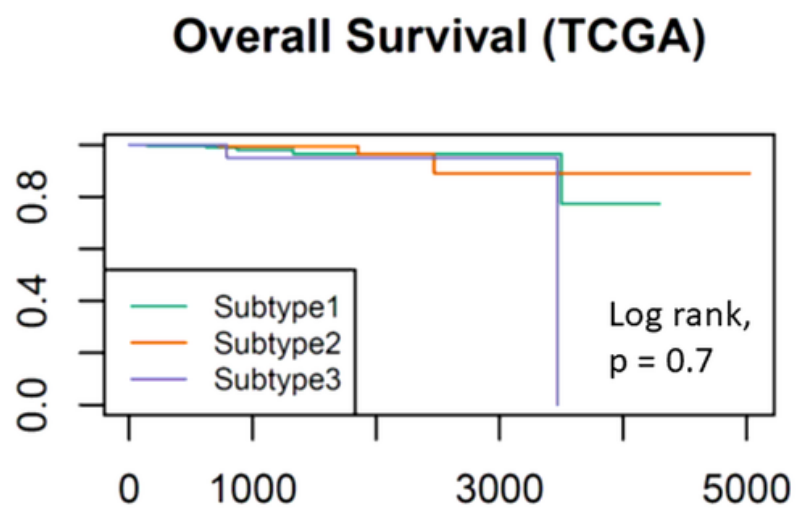

Days

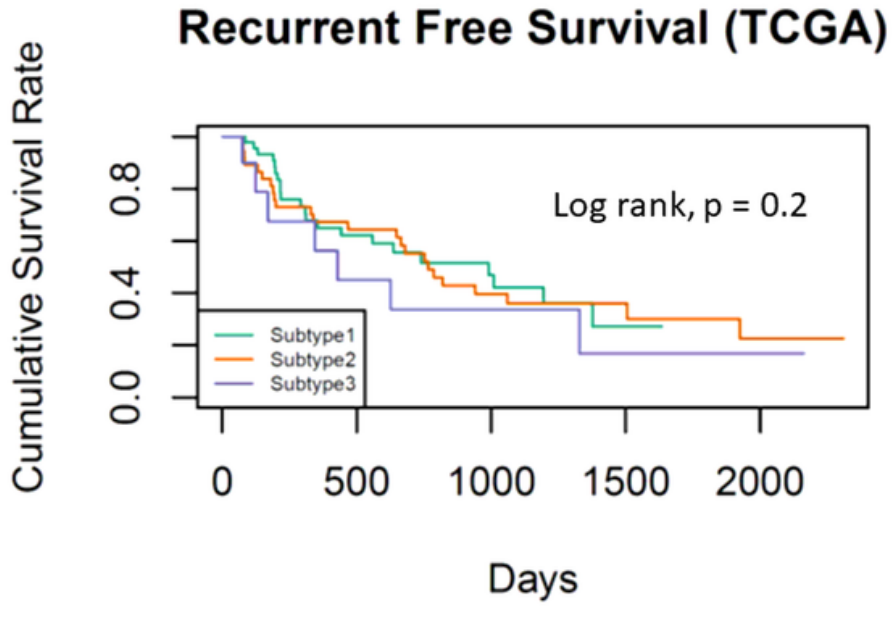

B

m6A Regulators Expression in GSE147493

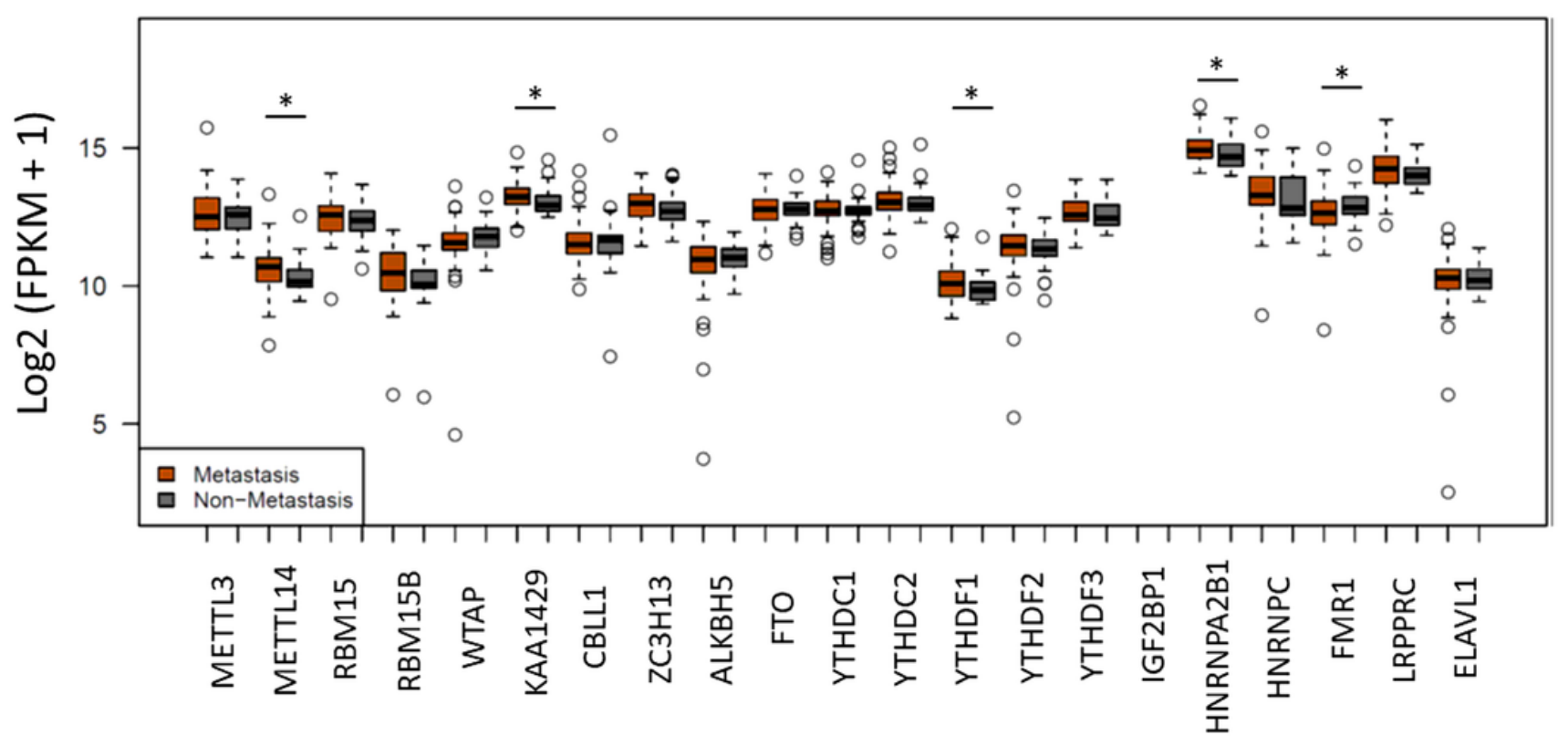

Figure 3

The regulators of $\mathrm{m} 6 \mathrm{~A}$ are more relevant to metastasis than recurrence in prostate cancer. A, Subtyping of prostate cancer by m6A regulators is not associated with patients' overall and recurrent-free survival (Log rank test, $p=0.7$ and 0.2 , respectively). $B, m 6 A$ regulators are associated with prostate cancer metastasis. Seven $\mathrm{m} 6 \mathrm{~A}$ regulators are differentially expressed between metastatic and non-metastatic prostate cancer. Student's t test. *, $\mathrm{p}<0.05$. 


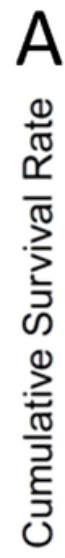
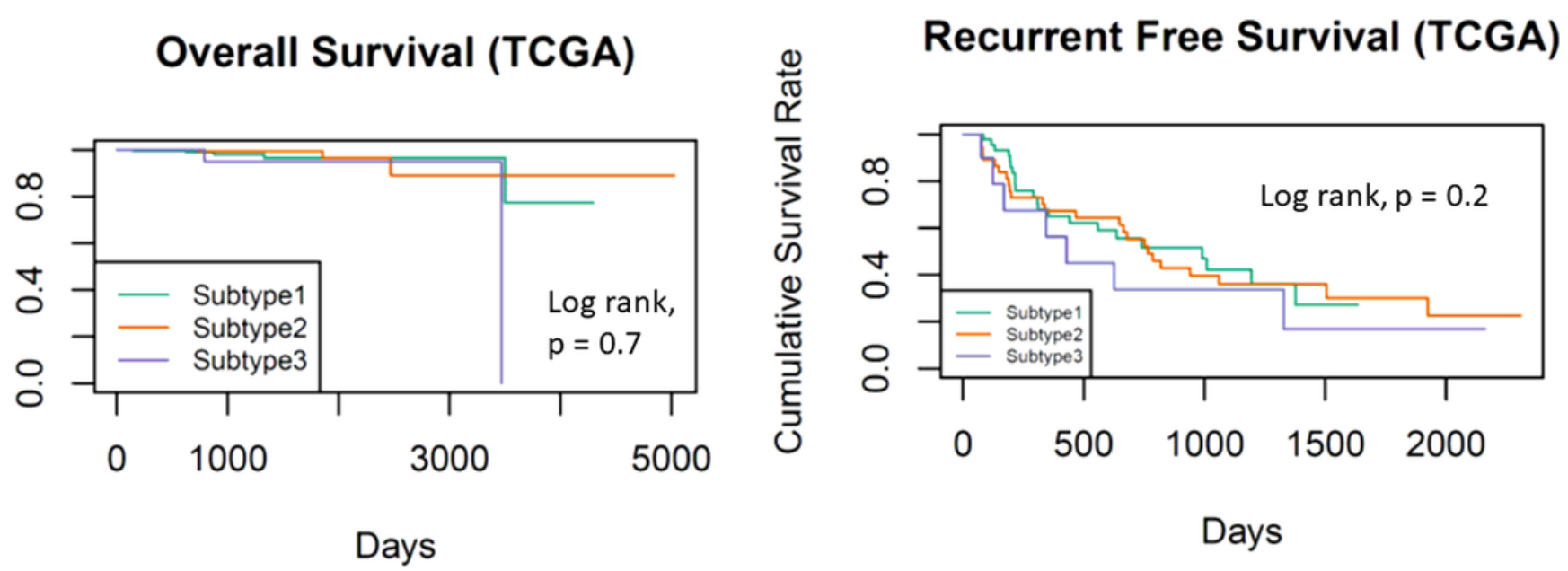

Days

B

m6A Regulators Expression in GSE147493

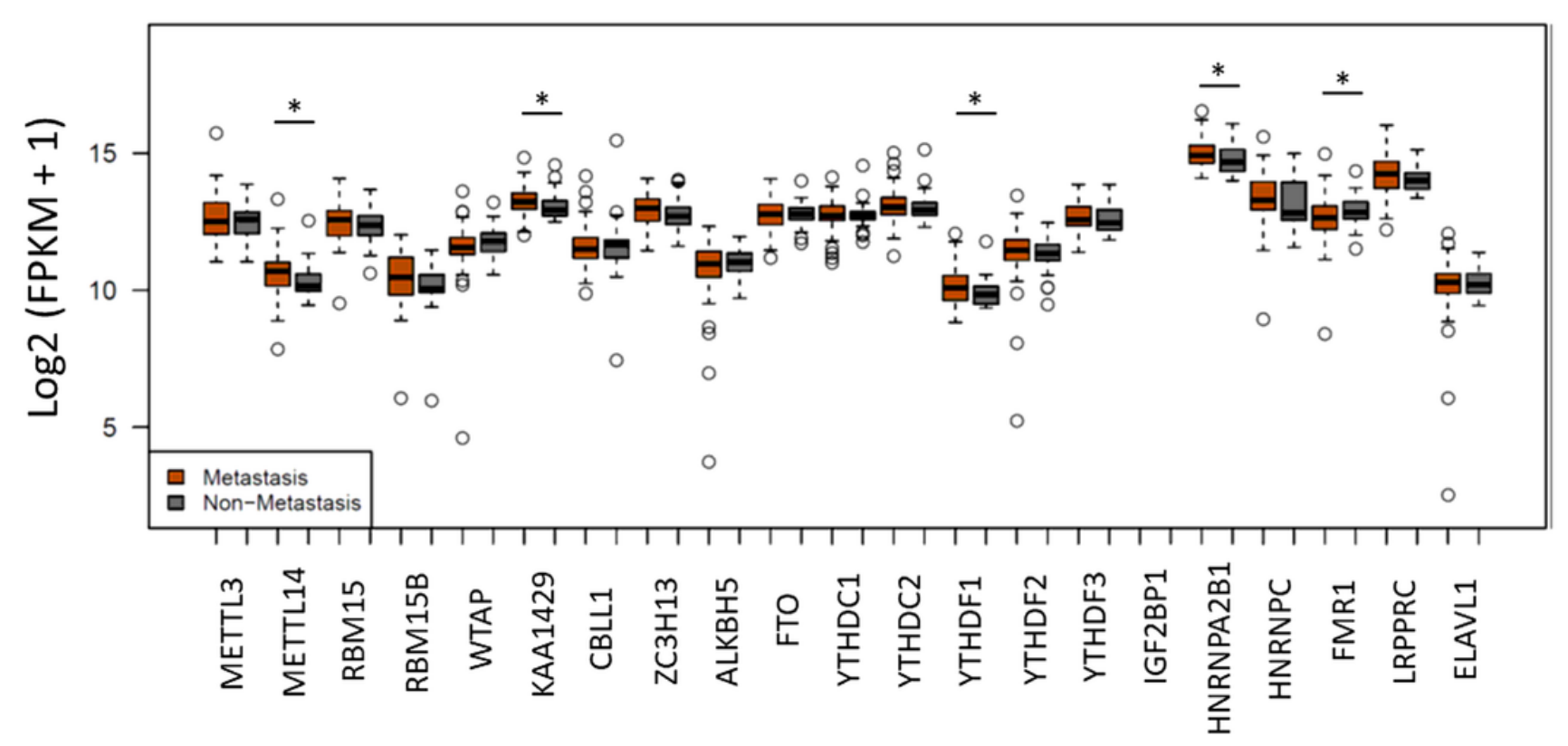

Figure 3

The regulators of $\mathrm{m} 6 \mathrm{~A}$ are more relevant to metastasis than recurrence in prostate cancer. A, Subtyping of prostate cancer by m6A regulators is not associated with patients' overall and recurrent-free survival (Log rank test, $\mathrm{p}=0.7$ and 0.2 , respectively). $\mathrm{B}, \mathrm{m} 6 \mathrm{~A}$ regulators are associated with prostate cancer metastasis. Seven $\mathrm{m} 6 \mathrm{~A}$ regulators are differentially expressed between metastatic and non-metastatic prostate cancer. Student's t test. *, $p<0.05$. 


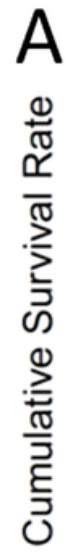

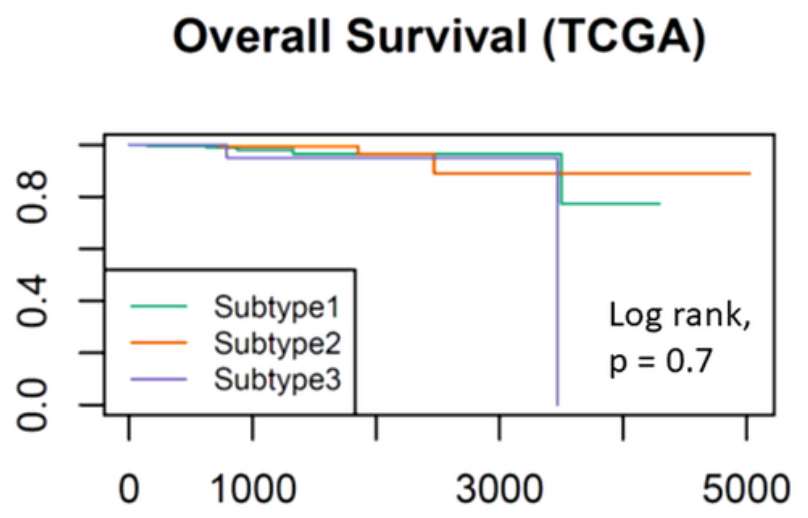

Days

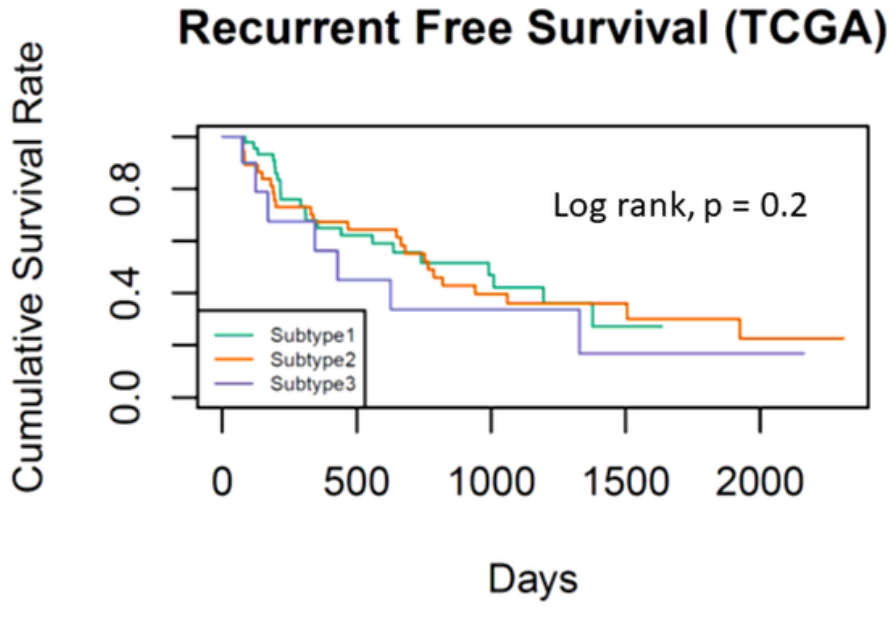

B

m6A Regulators Expression in GSE147493

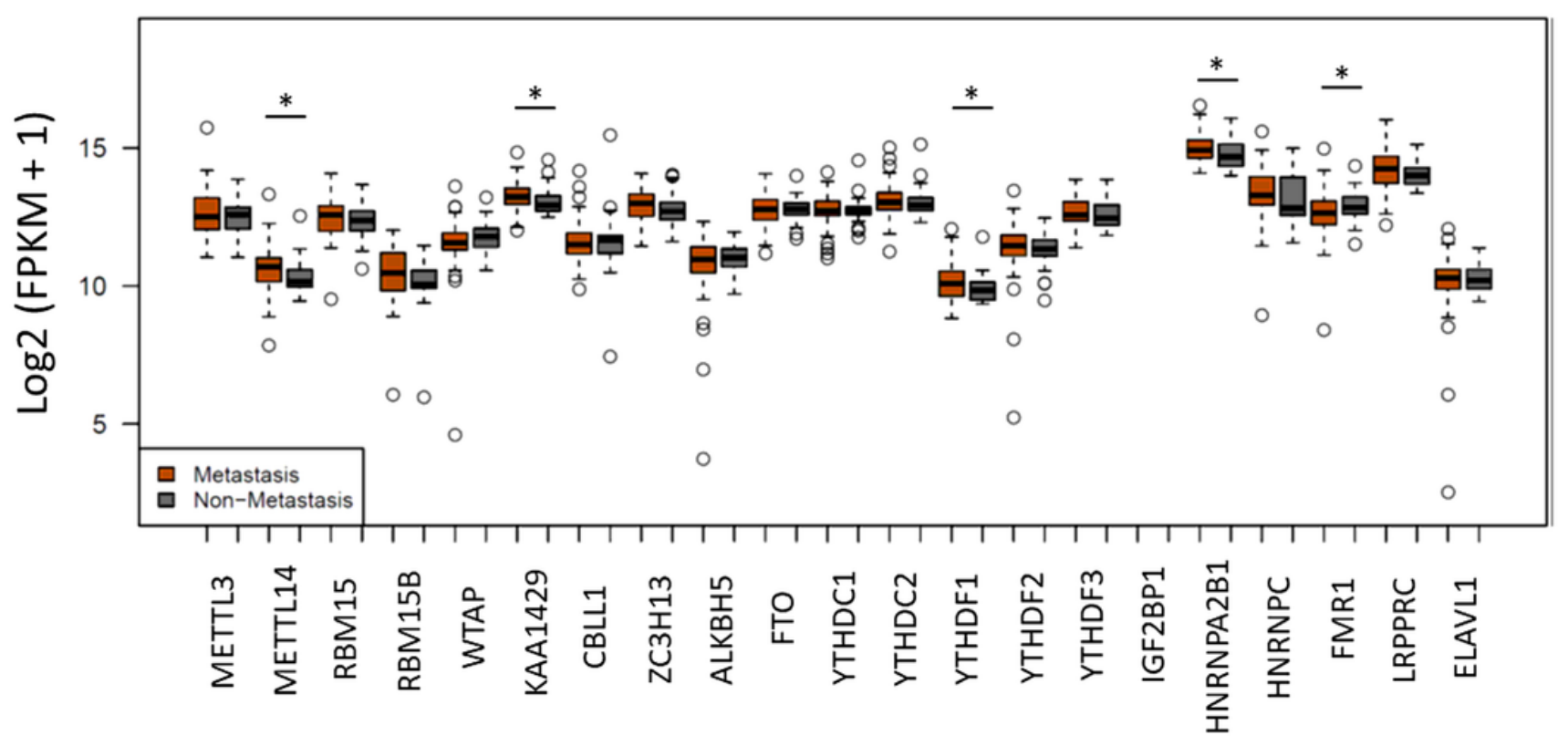

Figure 3

The regulators of $\mathrm{m} 6 \mathrm{~A}$ are more relevant to metastasis than recurrence in prostate cancer. A, Subtyping of prostate cancer by m6A regulators is not associated with patients' overall and recurrent-free survival (Log rank test, $p=0.7$ and 0.2 , respectively). $B, m 6 A$ regulators are associated with prostate cancer metastasis. Seven $\mathrm{m} 6 \mathrm{~A}$ regulators are differentially expressed between metastatic and non-metastatic prostate cancer. Student's t test. *, $\mathrm{p}<0.05$. 

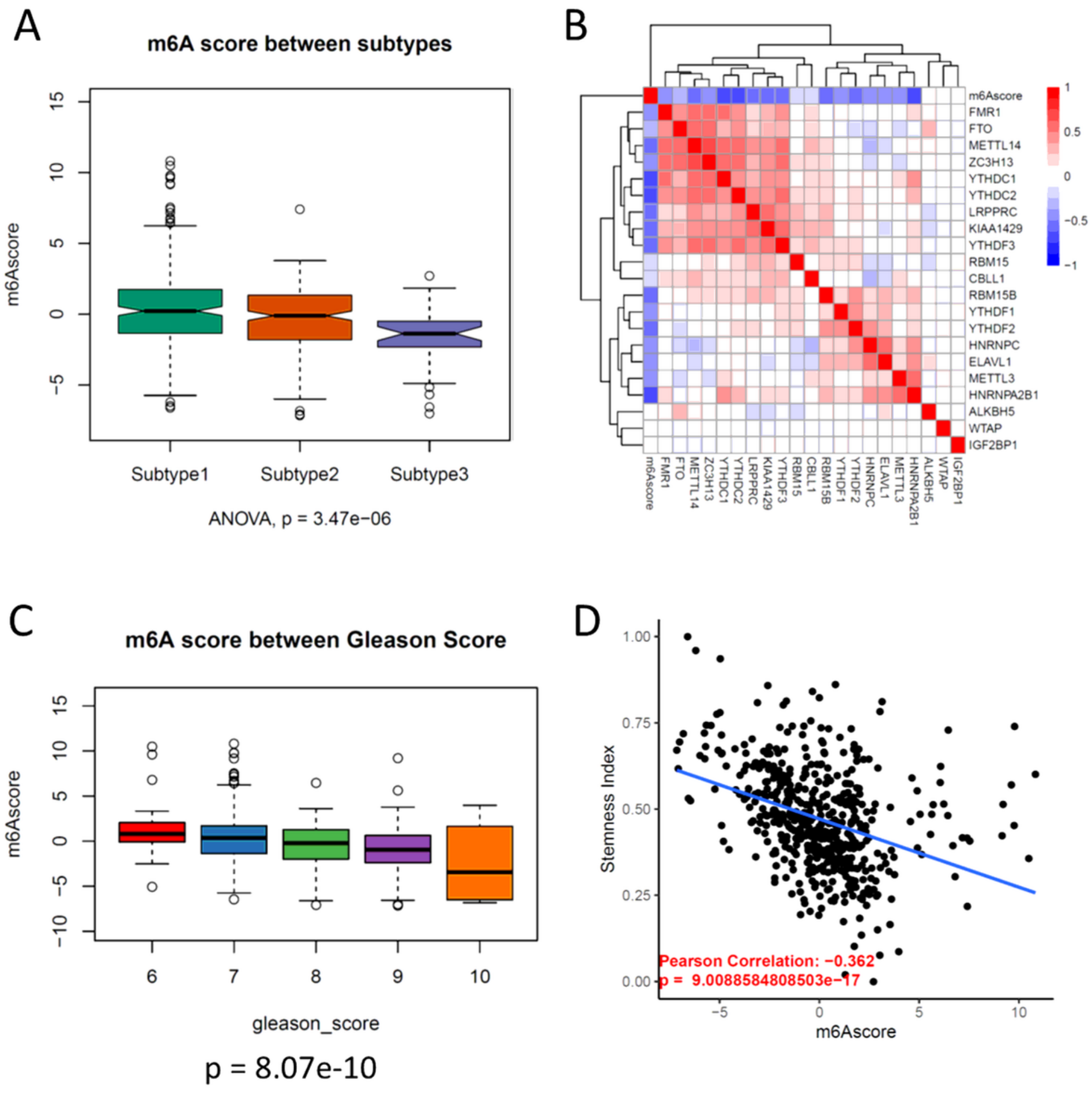

Figure 4

m6A score is related with Gleason Score and stemness score. A, Boxplot showing the different m6A scores between subtypes of m6A. Subtype 3 has the lowest m6A score. ANOVA, $p=3.47$ * $10-6$. B, Heatmap of the correlation between m6A regulators. The correlation coefficient is highlighted by colour. C, Boxplot showing that the m6A score is inversely associated with Gleason score (ANOVA, $p=8.07 * 10$ -10). Prostate cancer with higher Gleason Score has a lower m6A score. D, m6A score is inversely correlated with Stemness Index (Pearson Correlation, $r=-0.362 ; p=9.01 * 10-17$ ). 

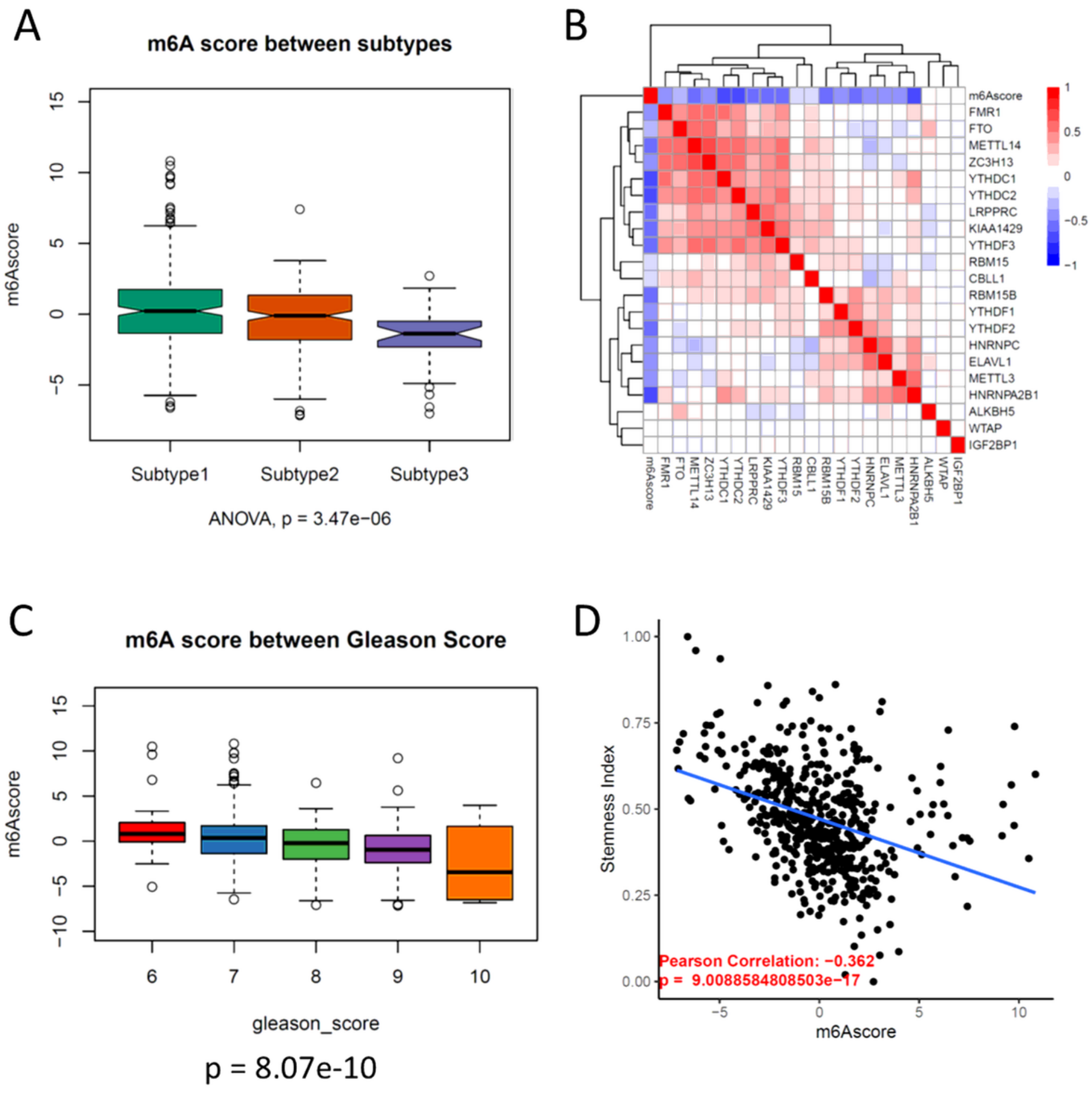

Figure 4

m6A score is related with Gleason Score and stemness score. A, Boxplot showing the different m6A scores between subtypes of m6A. Subtype 3 has the lowest m6A score. ANOVA, $p=3.47$ * $10-6$. B, Heatmap of the correlation between m6A regulators. The correlation coefficient is highlighted by colour. C, Boxplot showing that the m6A score is inversely associated with Gleason score (ANOVA, $p=8.07 * 10$ -10). Prostate cancer with higher Gleason Score has a lower m6A score. D, m6A score is inversely correlated with Stemness Index (Pearson Correlation, $r=-0.362 ; p=9.01 * 10-17$ ). 

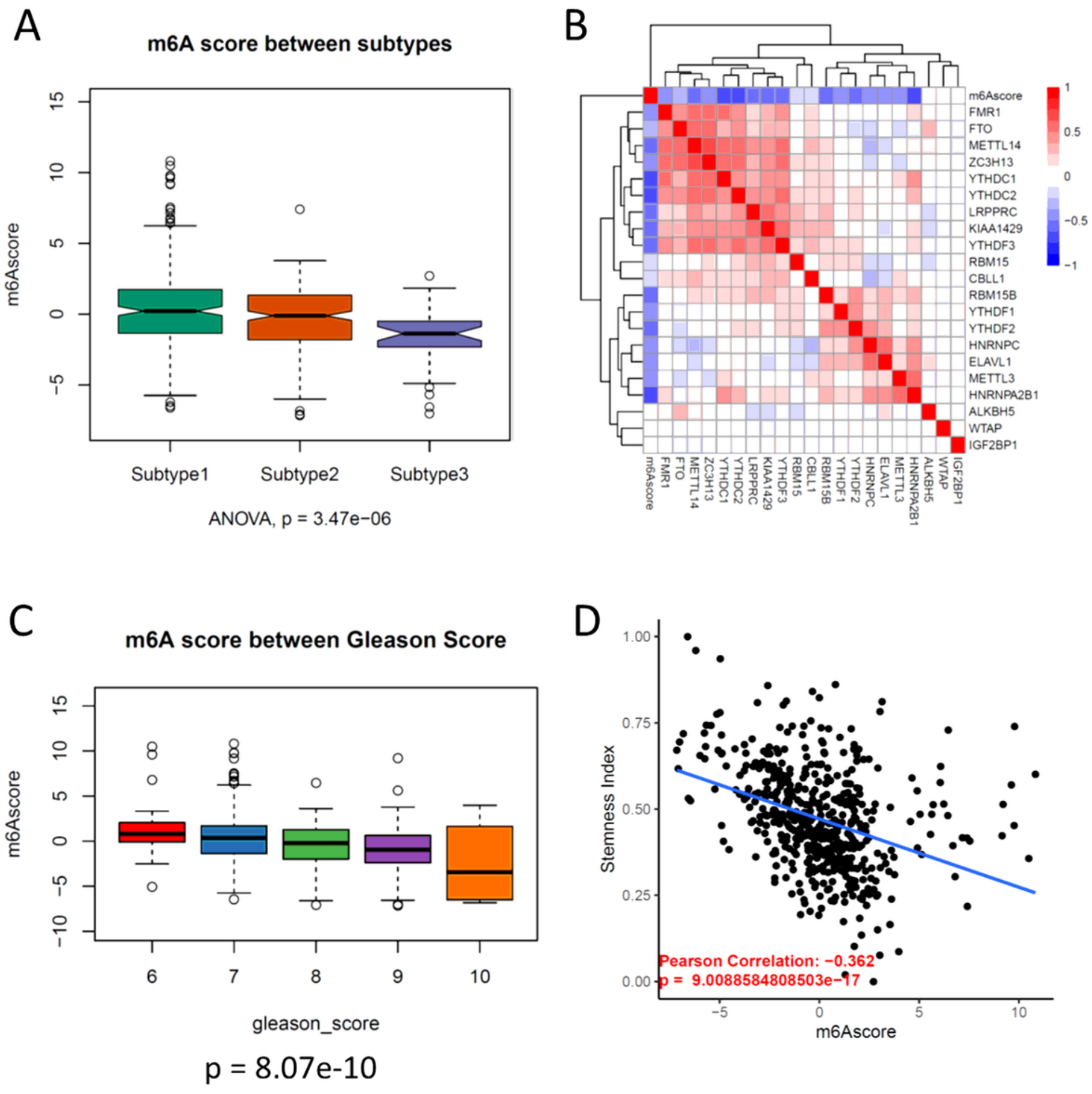

Figure 4

m6A score is related with Gleason Score and stemness score. A, Boxplot showing the different m6A scores between subtypes of m6A. Subtype 3 has the lowest m6A score. ANOVA, $p=3.47$ * $10-6$. B, Heatmap of the correlation between m6A regulators. The correlation coefficient is highlighted by colour. C, Boxplot showing that the m6A score is inversely associated with Gleason score (ANOVA, $p=8.07 * 10$ -10). Prostate cancer with higher Gleason Score has a lower m6A score. D, m6A score is inversely correlated with Stemness Index (Pearson Correlation, $r=-0.362 ; p=9.01 * 10-17$ ). 

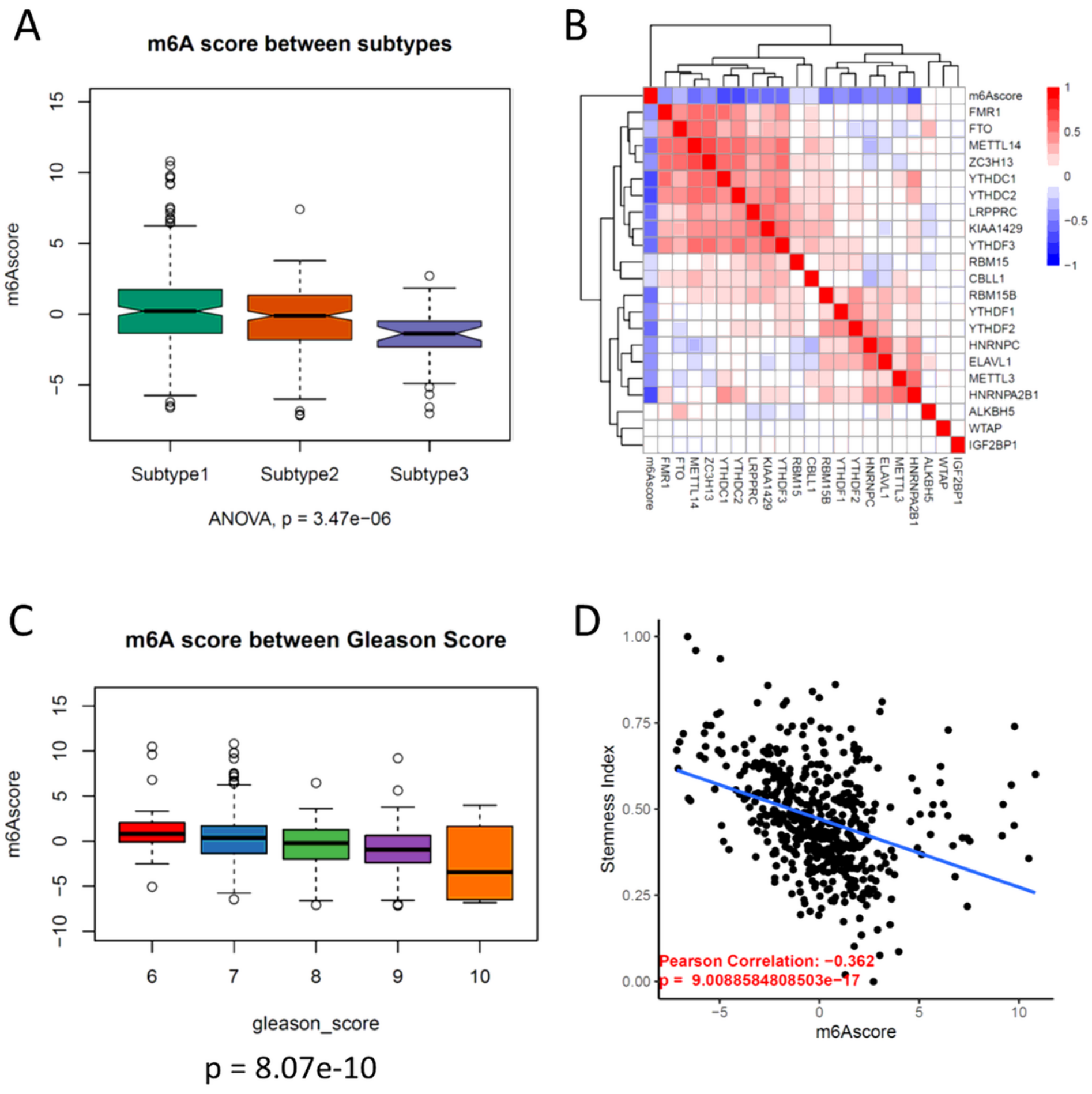

Figure 4

m6A score is related with Gleason Score and stemness score. A, Boxplot showing the different m6A scores between subtypes of m6A. Subtype 3 has the lowest m6A score. ANOVA, $p=3.47$ * $10-6$. B, Heatmap of the correlation between m6A regulators. The correlation coefficient is highlighted by colour. C, Boxplot showing that the m6A score is inversely associated with Gleason score (ANOVA, $p=8.07 * 10$ -10). Prostate cancer with higher Gleason Score has a lower m6A score. D, m6A score is inversely correlated with Stemness Index (Pearson Correlation, $r=-0.362 ; p=9.01 * 10-17$ ). 
A

m6A Score (m6A.reg.ov) in GSE147493

B

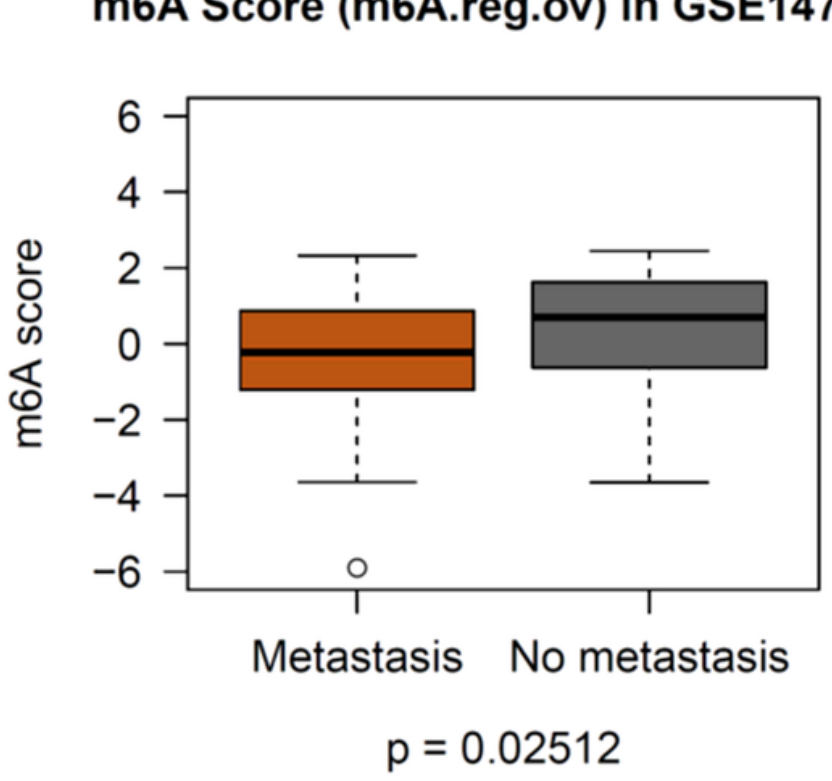

ROC for prediction of recurrence by $\mathrm{m} 6 \mathrm{~A}$ si

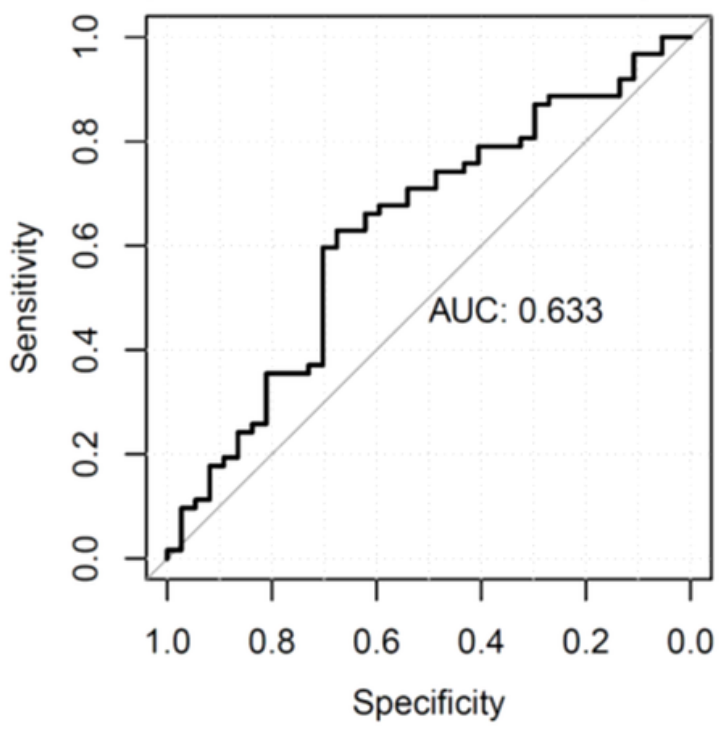

C

m6A Score in GSE6919

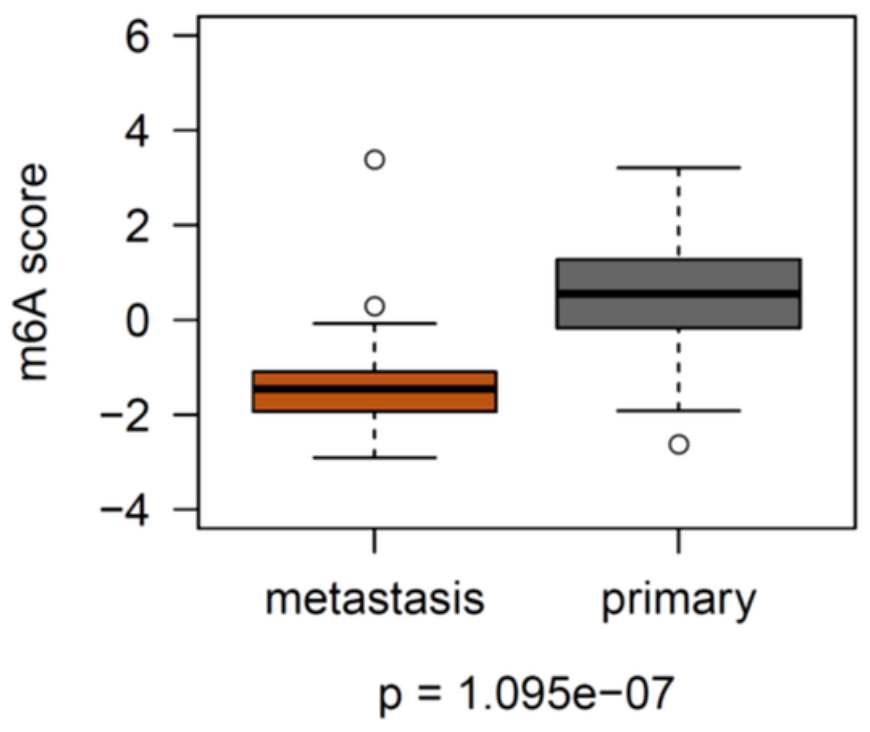

D

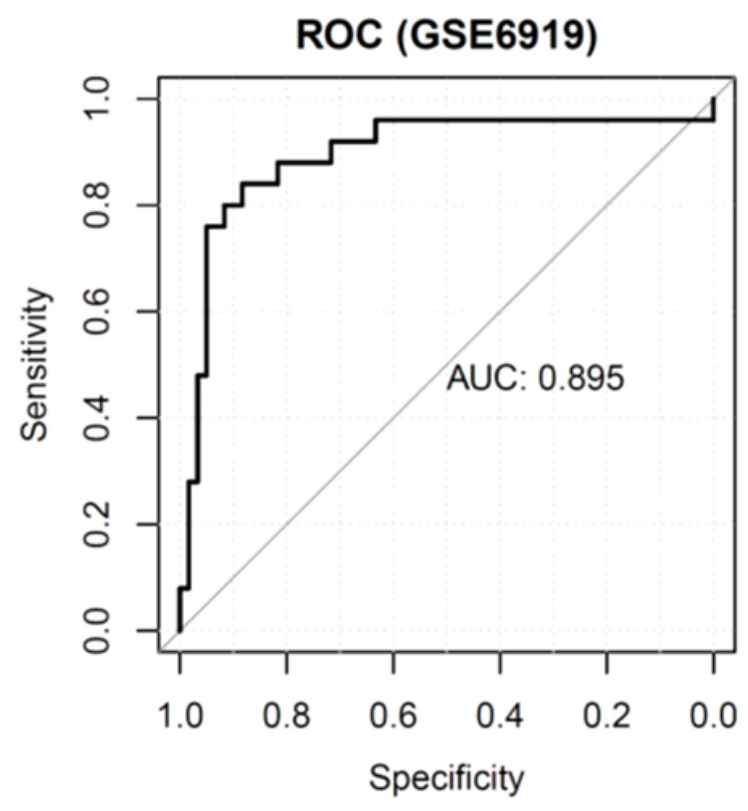

Figure 5

The m6A regulators are differentially expressed between metastatic and non-metastatic prostate cancer. $\mathrm{A}$, Boxplot showing metastatic prostate cancer has a significantly lower m6A score. Student $t$ test, $p=$ 0.02512. B, m6A score showed a moderate discrimination of metastatic prostate cancer from nonmetastasis. The accuracy of the m6A score is determined by the Area Under the Curve (AUC). The effectiveness of m6A score is validated in another independent cohort (GSE6919). The boxplot (C) showing the metastatic PCa has significantly lower expression of m6A score than primary PCa. D, ROC of 
GSE6919 dataset demonstrating the accuracy of prediction to metastatic PCa is $89.5 \%$ in the validation cohort.

A

m6A Score (m6A.reg.ov) in GSE147493

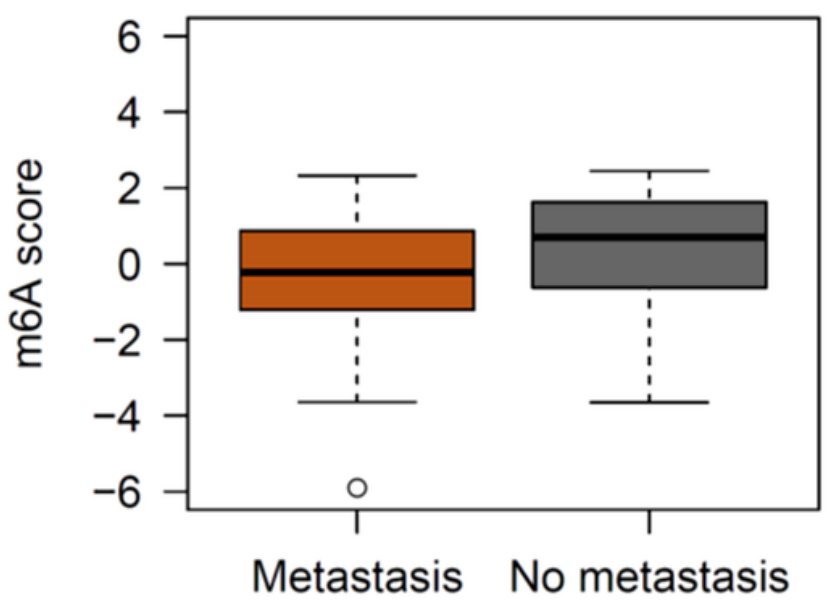

$p=0.02512$

B

ROC for prediction of recurrence by m6A si

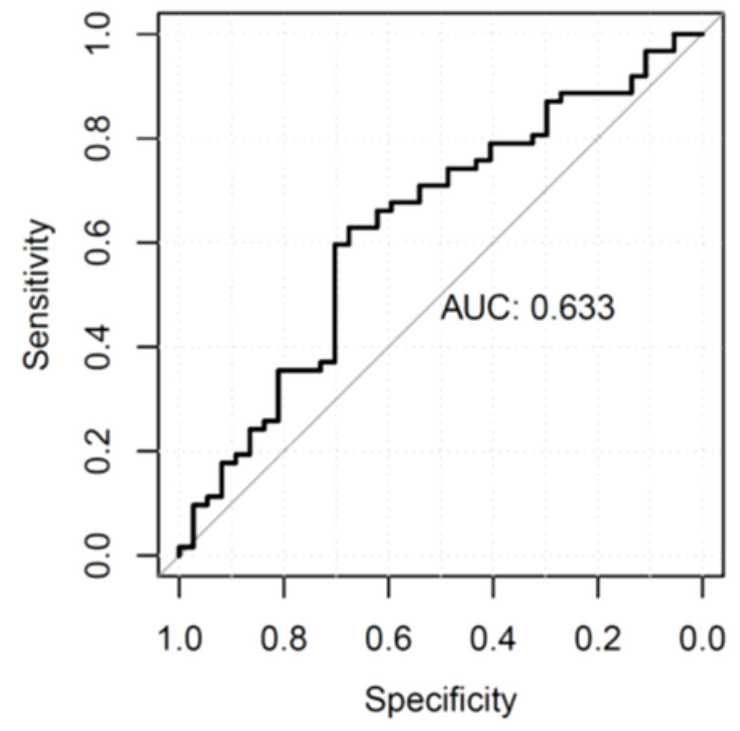

D

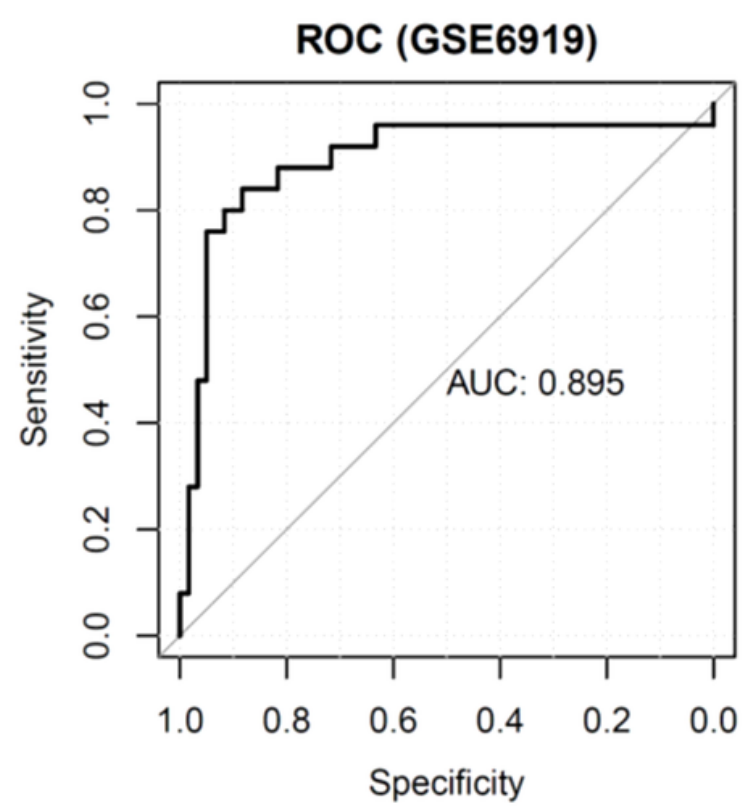

Figure 5

The m6A regulators are differentially expressed between metastatic and non-metastatic prostate cancer. $\mathrm{A}$, Boxplot showing metastatic prostate cancer has a significantly lower m6A score. Student $t$ test, $p=$ 0.02512. B, m6A score showed a moderate discrimination of metastatic prostate cancer from nonmetastasis. The accuracy of the m6A score is determined by the Area Under the Curve (AUC). The 
effectiveness of m6A score is validated in another independent cohort (GSE6919). The boxplot (C) showing the metastatic PCa has significantly lower expression of m6A score than primary PCa. D, ROC of GSE6919 dataset demonstrating the accuracy of prediction to metastatic PCa is $89.5 \%$ in the validation cohort.

A

B

m6A Score (m6A.reg.ov) in GSE147493

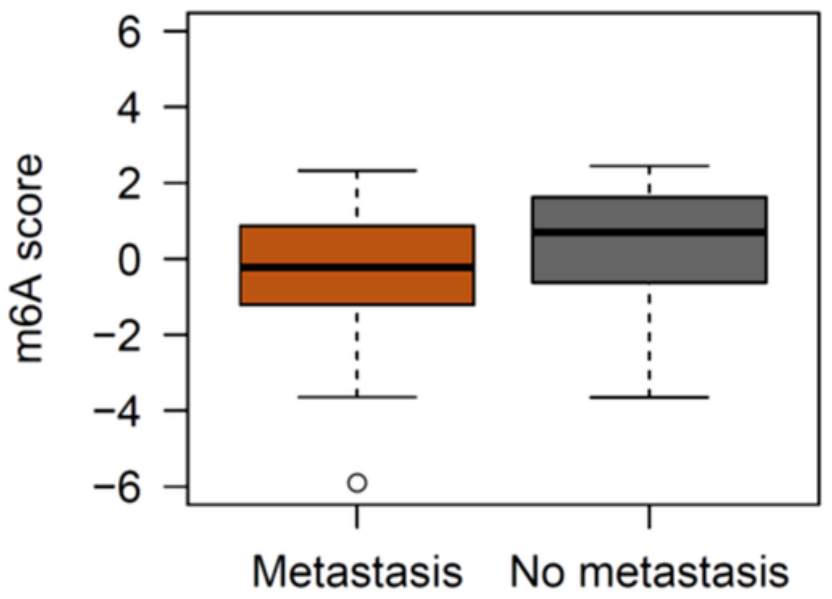

$p=0.02512$

ROC for prediction of recurrence by $\mathrm{m} 6 \mathrm{~A}$ si

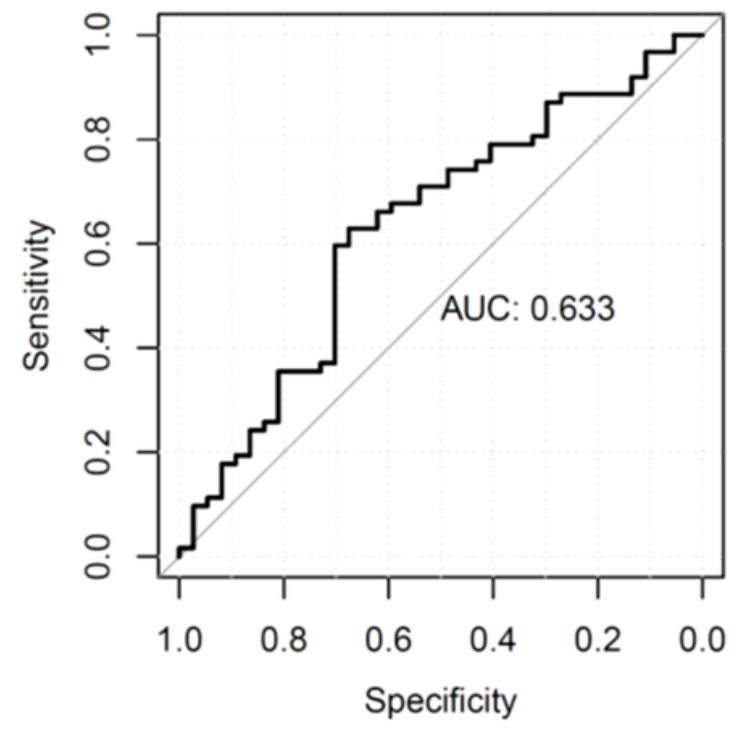

D

m6A Score in GSE6919

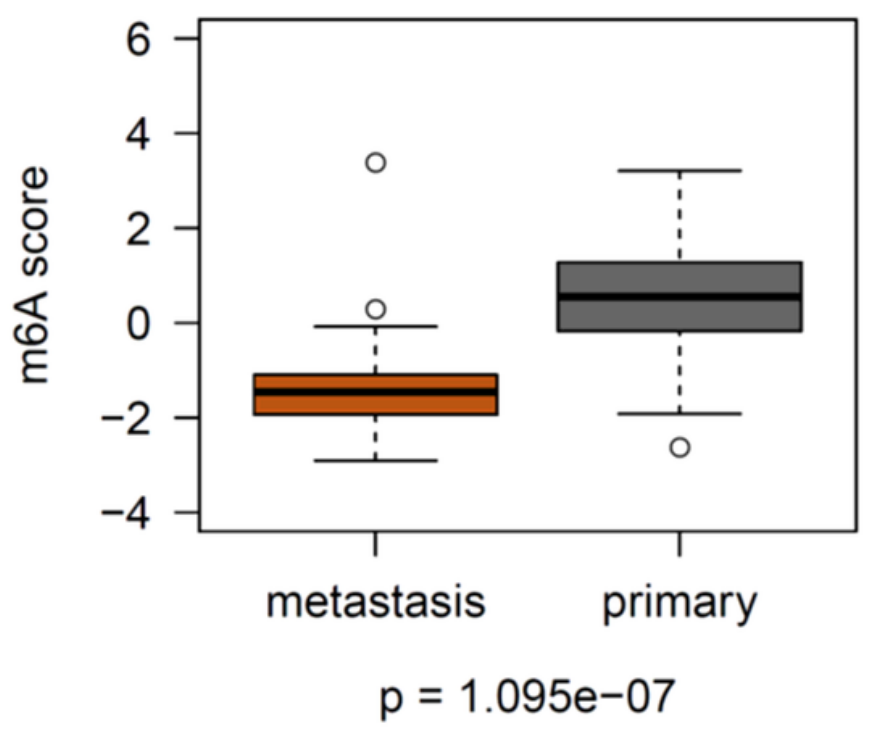

ROC (GSE6919)

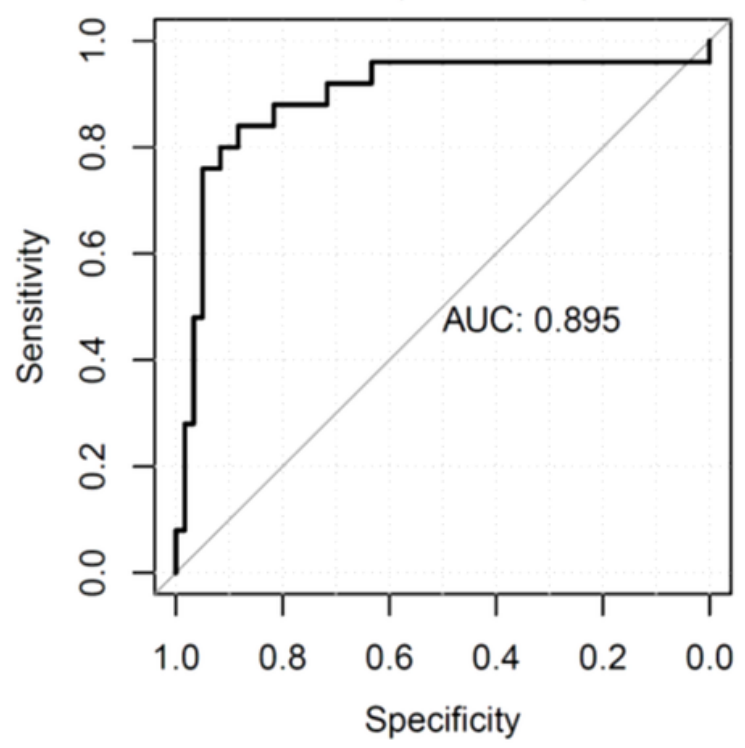

Figure 5

The m6A regulators are differentially expressed between metastatic and non-metastatic prostate cancer. $\mathrm{A}$, Boxplot showing metastatic prostate cancer has a significantly lower m6A score. Student $t$ test, $p=$ 
0.02512. B, m6A score showed a moderate discrimination of metastatic prostate cancer from nonmetastasis. The accuracy of the m6A score is determined by the Area Under the Curve (AUC). The effectiveness of m6A score is validated in another independent cohort (GSE6919). The boxplot (C) showing the metastatic PCa has significantly lower expression of m6A score than primary PCa. D, ROC of GSE6919 dataset demonstrating the accuracy of prediction to metastatic PCa is $89.5 \%$ in the validation cohort.

A

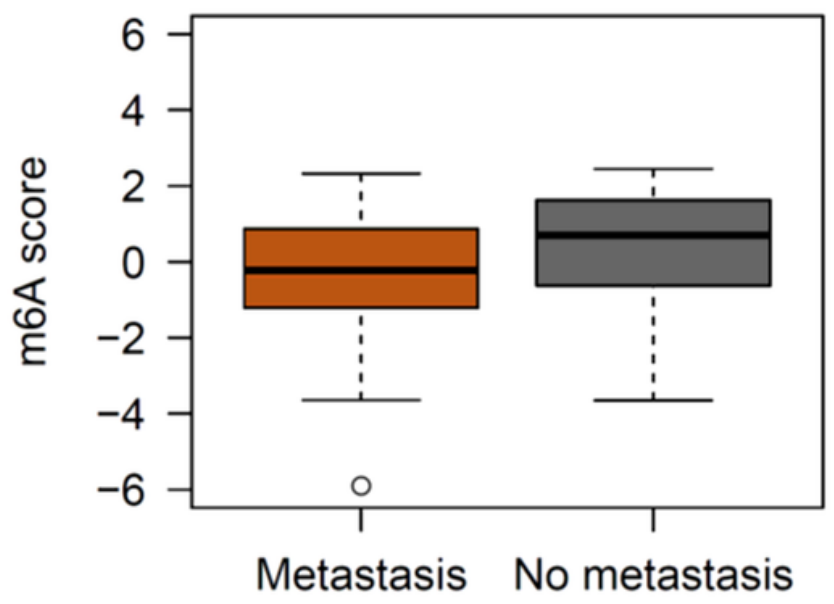

$p=0.02512$

C

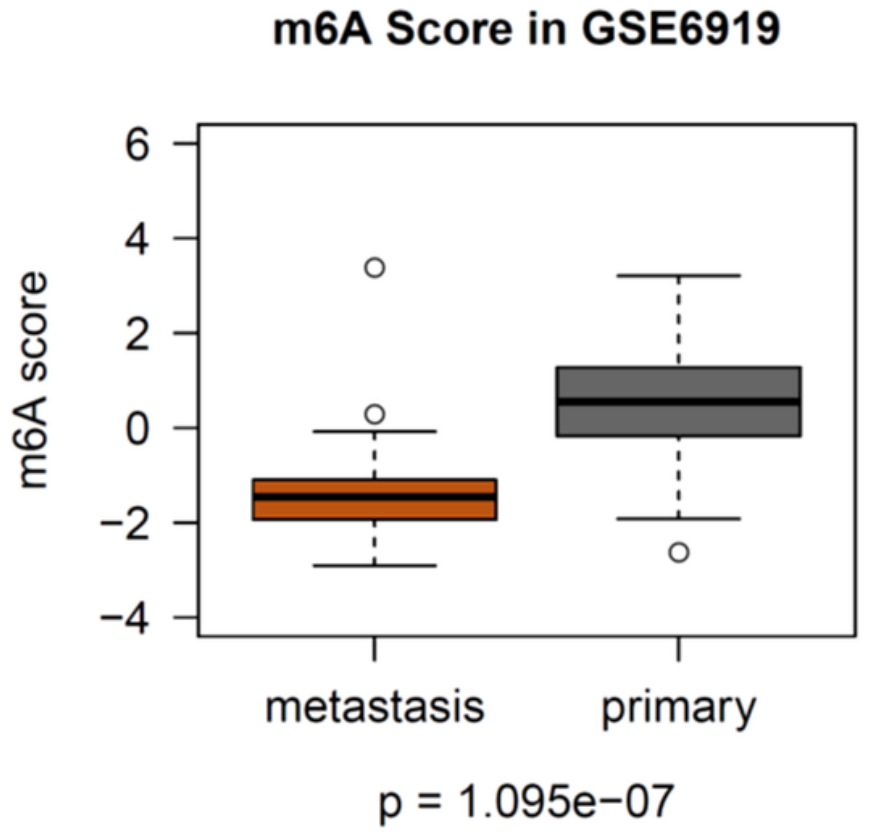

B

ROC for prediction of recurrence by $\mathrm{m} 6 \mathrm{~A}$ si

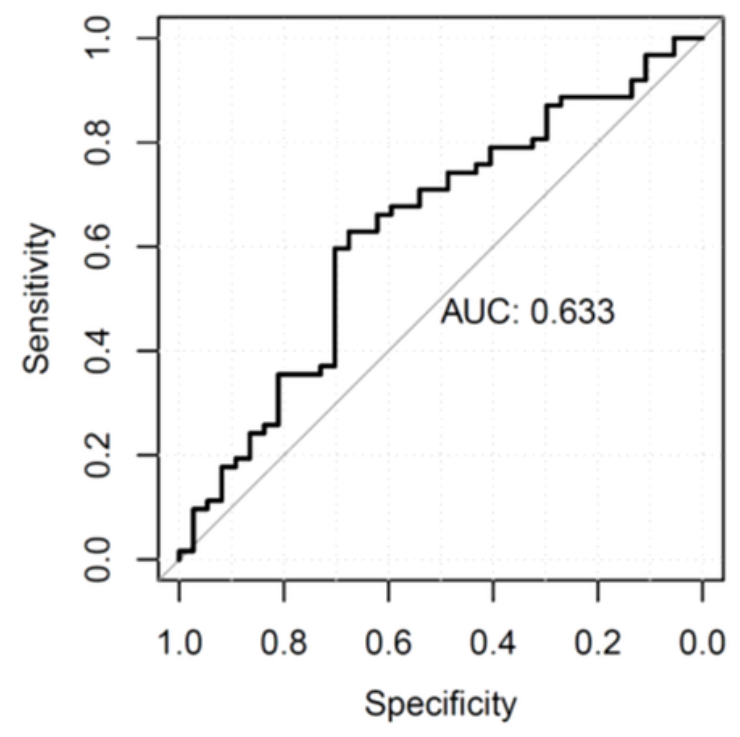

D

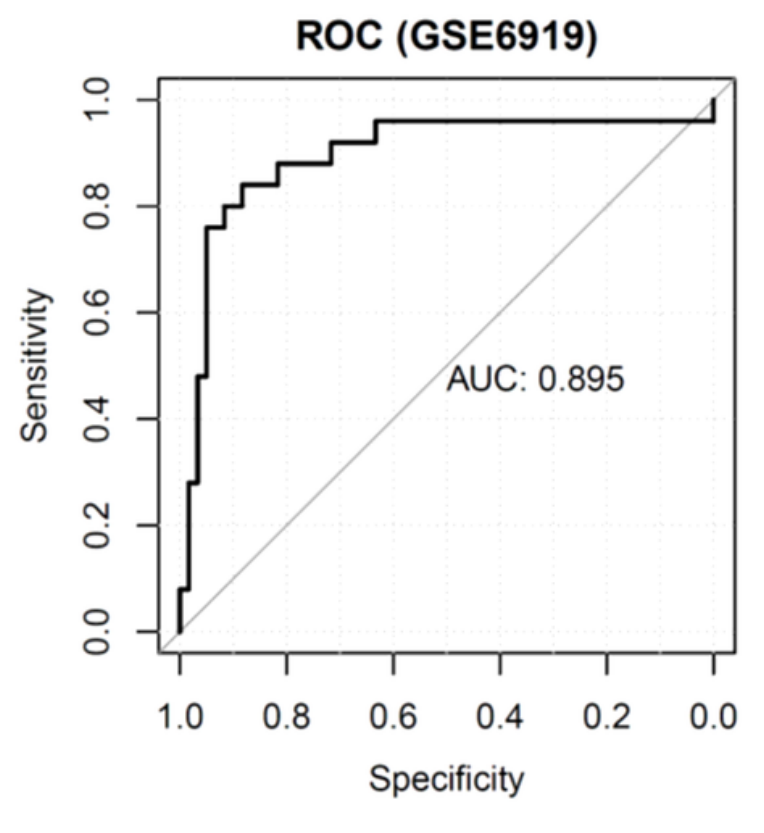

Figure 5 
The m6A regulators are differentially expressed between metastatic and non-metastatic prostate cancer. $\mathrm{A}$, Boxplot showing metastatic prostate cancer has a significantly lower m6A score. Student $t$ test, $p=$ 0.02512. B, m6A score showed a moderate discrimination of metastatic prostate cancer from nonmetastasis. The accuracy of the m6A score is determined by the Area Under the Curve (AUC). The effectiveness of m6A score is validated in another independent cohort (GSE6919). The boxplot (C) showing the metastatic PCa has significantly lower expression of m6A score than primary PCa. D, ROC of GSE6919 dataset demonstrating the accuracy of prediction to metastatic PCa is $89.5 \%$ in the validation cohort.

\section{Supplementary Files}

This is a list of supplementary files associated with this preprint. Click to download.

- supple8.tif

- supple8.tif

- supple8.tif

- supple8.tif

- supple7.tif

- supple7.tif

- supple7.tif

- supple7.tif

- supple6.tif

- supple6.tif

- supple6.tif

- supple6.tif

- supple5.tif

- supple5.tif

- supple5.tif

- supple5.tif

- supple3.tif

- supple3.tif

- supple3.tif

- supple3.tif

- supple2.tif

- supple2.tif

- supple2.tif 
- supple2.tif

- supple1.tif

- supple1.tif

- supple1.tif

- supple1.tif

- Table1.xIsx

- Table1.xIsx

- Table1.xlsx

- Table1.xIsx 